\title{
Nonlinear Control of Feedforward Systems With Bounded Signals
}

\author{
Georgia Kaliora and Alessandro Astolfi
}

\begin{abstract}
The stabilization problem for a class of nonlinear feedforward systems is solved using bounded control. It is shown that when the lower subsystem of the cascade is input-to-state stable and the upper subsystem not exponentially unstable, global asymptotic stability can be achieved via a simple static feedback having bounded amplitude that requires knowledge of the "upper" part of the state only. This is made possible by invoking the bounded real lemma and a generalization of the small gain theorem. Thus, stabilization is achieved with typical saturation functions, saturations of constant sign, or quantized control. Moreover, the problem of asymptotic stabilization of a stable linear system with bounded outputs is solved by means of dynamic feedback. Finally, a new class of stabilizing control laws for a chain of integrators with input saturation is proposed. Some robustness issues are also addressed and the theory is illustrated with examples on the stabilization of physical systems.
\end{abstract}

Index Terms-Bounded control, bounded-real lemma, forwarding, nonlinear stabilization.

\section{INTRODUCTION}

$\mathbf{I}$ N THIS paper, we study the problem of asymptotic stabilization with bounded control of stable cascades described by equations of the form

$$
\begin{aligned}
\dot{z} & =J z+h(\xi)+r(\xi) u \\
\dot{\xi} & =f(\xi)+g(\xi) u
\end{aligned}
$$

and some related problems. See Section II for the precise formulation and the standing assumptions.

Nonlinear control with saturated signals is a problem that although well studied (see, e.g., [10], [33], [35], [32], and the references therein) still gathers a lot of interest [9], [18], [19], [22]. Limitations on available energy impose bounded input signals, while it is also very common that due to sensors limitations the outputs of the system are bounded. System (1) belongs to the family of systems in feedforward form. This class of systems can be stabilized using the forwarding approach or one of its modifications; see e.g., [10], [29], [21], [25], and [2]. On the other hand, nonlinear small gain theorem based approaches have also been used for the stabilization of these systems [36], [33], [18], [1]. Finally, (1) can (under some special assumptions) also be studied from an absolute stability [38] point of view.

Manuscript received October 23, 2003; revised March 17, 2004 and May 4, 2004. Recommended by Associate Editor Z. Lin. This work was supported by the TMR Network, NACO 2, and the State Scholarships Foundation of Greece.

The authors are with the Department of Electrical and Electronic Engineering, Imperial College, London SW7 2BT, U.K. (e-mail: g.kaliora@imperial.ac.uk; a.astolfi@imperial.ac.uk).

Digital Object Identifier 10.1109/TAC.2004.837572
Forwarding is a systematic tool for the stabilization of general cascades, a special case of which is the form described by (1). This methodology requires, in general, the (approximate) solution of a partial differential equation and tends to generate complex control laws. Moreover, although forwarding tackles successfully saturated inputs, it is not a low amplitude design, so it does not impose restrictions on the control amplitude. The control Lyapunov function approach can also provide control laws for stabilization in the presence of input constraints, with the use of universal formulae [15]. Relevant results are general, however, large amount of the studies on low amplitude designs is typically based on small gain considerations. They also require full state feedback, and in some cases only semiglobal results are provided [17], [32], [7], [34], [27].

From a structural point of view, for systems described by a generalized linear [33], [35], [17] or nonlinear [18], [9] chain of integrators, the control laws consist of a generalization of the nested saturations scheme of [35] or linear combinations of saturations [33]. These designs also make use of passivity, in the sense that, at each step of the procedure, the feedback consists of a function of the state for which is a relative degree one output. See also [1], where Teel's nested saturation scheme is robustified against unmodeled dynamics. On the other hand, when linear versions of system (1) are considered, an analysis based on absolute stability can be easily implemented and can lead to simple control laws. This way of thinking also provides flexibility and robustness against some classes of perturbations. This is made possible, because unlike with passivity based designs, no phase restriction is imposed.

The results of this paper are motivated by the observation that under the assumption that the lower subsystem of (1) is input-to-state stable (ISS) ${ }^{1}$ and locally exponentially stable, an absolute stability point of view can be used in the design of stabilizing saturated controllers for linear as well as nonlinear systems. In a more precise formulation, the results stem from a general result on the $L_{2}$ stability of feedback interconnections found in [26] and from the linear bounded real lemma [8]. The proposed design requires partial state feedback only, and it bears no connection to passivity arguments. As a matter of fact, it will be shown that, in a very clear and natural framework, a number of stabilization issues for (1), such as global asymptotic and ISS stabilization (possibly with restrictions), robust stabilization, and stabilization with bounded outputs can be addressed.

More specifically, the main contribution of this paper is the presentation of new class of bounded control laws for (1).

\footnotetext{
${ }^{1}$ This condition can be relaxed to ISS with restrictions or to global asymptotic stability.
} 
Following this general result, the linear bounded real lemma and the generalized small gain theorem of [26] are used to solve in a unified way the following problems.

- Robust stabilization of a particular class of systems (1) with partial state feedback in the presence of time delays. Under the present framework, these perturbations can be accommodated in a natural way, unlike the case where passivity-based controllers are used. See, for example, [20], where some robustness issues of the nested saturations scheme have been studied.

- Asymptotic stabilization with control of constant sign.

- Practical stabilization with quantized control, i.e., it will be shown that a control input taking values in a discrete set can drive the state of the closed loop system in an arbitrarily small neighborhood of the origin.

In addition, the problem of global asymptotic stabilization for stable linear systems with bounded output is solved via dynamic linear feedback. Stabilization with feedback of perturbed and bounded outputs was achieved via time varying control in [6], [14], and [23], and via dynamic control, that includes state observation, in [16] and [22]. The dynamic law presented here is not based on state estimation and it is applicable to minimum and nonminimum phase systems, providing a partial answer to the question raised in [16] about the stabilizability of output feedback systems with unstable zeros in the presence of saturated outputs.

Another byproduct of the main result is a new globally asymptotically stabilizing control law for a chain of integrators in the presence of input saturation which is obtained with recursive application of the main result. This is conceptually and structurally different from the ones of [35], [33], [18], and [9]. Moreover, the stabilization of mechanical systems is addressed as an application of the main results. In particular, the translational oscillator with a rotational actuator (TORA) [3], is globally asymptotically stabilized by output feedback. Various constructive nonlinear control methodologies have been tested on this system (see, for example, [11], [22], and [29]), while in [24] the problem was set in a Euler-Lagrange framework and a passivity based output feedback controller was proposed. With the exception of this last reference, all proposed stabilizing controllers either require full state feedback or utilize some kind of state observer. In this paper, it will be shown that a simple dynamic output feedback controller of dimension one can globally asymptotically stabilize the TORA, which may be compared with the elaborate stabilization scheme of [11]. Also, a preliminary result on the stabilization of underactuated ships moving on a linear course is presented.

As mentioned previously, the majority of the results that are presented in this paper are established from an interconnections point of view, i.e., they are proven with the application of a generalization of the small gain theorem. However, all of them can be phrased in Lyapunov stability and invariance principle arguments.

In what follows, the construction of the bounded control signals-or the mathematical description of a bounded output-will be achieved with the use of saturation functions. More specifically, we will use three different types of such nonlinearities, all belonging to the sector $[0,1],{ }^{2}$ the simplest of which, denoted with $\sigma_{s}(\cdot), \sigma_{+}(\cdot)$, and $\sigma_{q}(\cdot)$, are

$$
\begin{aligned}
& \sigma_{s}(y)=\operatorname{sign}(y) \min \{|y|, 1\} \quad \sigma_{+}(y)=\max \left\{0, \sigma_{s}(y)\right\} \\
& \sigma_{q}(y)= \begin{cases}0, & \text { for }|y|<1 \\
\operatorname{sign}(y), & \text { elsewhere. }\end{cases}
\end{aligned}
$$

For the rest of this paper, we use the general symbol $\sigma(\cdot)$ to denote any of the functions (2).

The paper is organized as follows. In Section II, we present a preliminary result on the stabilization of a cascade consisting of an ISS-locally exponentially stable (LES) system driving an integrator. In the same section we formulate precisely the two main problems that are addressed and solved. In Section III, we present and prove two useful Lemmas about the solvability of a matrix inequality. In Section IV, we elaborate on our main result on the stabilization of nonlinear feedforward systems with a bounded, partial state feedback control law. Motivated by the results in Sections III and IV, a dynamic control law that solves the problem of asymptotic stabilization of a linear stable single-input-singleoutput (SISO) system with bounded output is presented in Section V. In Section VI, we give some applications of our main result. Finally, in Section VII, we provide some conclusions.

Comment: With the exception of the saturation functions defined in (2), it is assumed that all mappings and functions are at least $\mathcal{C}^{1}$, throughout the paper. Note that the saturation functions (2) are piecewise $\mathcal{C}^{1}$. Moreover, whenever linear approximations are used these are always considered at the origin and for functions and mappings that are $\mathcal{C}^{1}$ at the origin. It will become clear that all statements that involve $\sigma_{s}(\cdot)$ can be applied iteratively.

Notation: The symbol $\|s\|$ is used to denote the Euclidean norm of a vector $s$.

\section{Motivating Result AND PROBLEM Formulation}

In this section, we show how stabilization of a simple cascade can be obtained using bounded partial state feedback, and we state formally the problems studied in this paper. Consider a system described by equations of the form

$$
\begin{aligned}
& \dot{z}=h(\xi) \\
& \dot{\xi}=f(\xi)+g(\xi) u
\end{aligned}
$$

with $z \in \mathbb{R}, \xi \in \mathbb{R}^{n}$, and $u \in \mathbb{R}$ and assume that the lower subsystem is LES and ISS with respect to $u$. We now show that this cascaded system can be stabilized with a simple bounded feedback law that requires knowledge of $z$ only. The rationale behind this result is straightforward. To begin with note that if $|u| \leq \epsilon$ is sufficiently small, by LES and ISS of the $\xi$-subsystem, any trajectory of the closed loop system will converge to the slice $\|\xi\|<\delta_{\epsilon}$, where $\delta_{\epsilon}$ can be made arbitrarily small reducing $\epsilon$. Note also that $z(t)$ is bounded for all bounded $t$. On the slice $\|\xi\|<\delta_{\epsilon}$ the system can be approximated by a linear time invariant system given by the equations

$$
\left[\begin{array}{c}
\dot{z} \\
\dot{\xi}
\end{array}\right]=\left[\begin{array}{ll}
0 & H \\
0 & F
\end{array}\right]\left[\begin{array}{l}
z \\
\xi
\end{array}\right]+\left[\begin{array}{c}
0 \\
G
\end{array}\right] u
$$

${ }^{2} \mathrm{~A}$ function $\sigma(y)$ is said to belong to the sector $\left[k_{1}, k_{2}\right]$ if for all $k_{1} \leq$ $\sigma(y) / y \leq k_{2}$. 
where

$$
\left.\left.H \triangleq \frac{\partial h(\xi)}{\partial \xi}\right|_{\xi=0} F \triangleq \frac{\partial f(\xi)}{\partial \xi}\right|_{\xi=0} G \triangleq g(0)
$$

Then, the following result can be established.

Proposition 1: Consider (3) with $f(0)=0$ and $h(0)=0$, and the nonlinearity $\sigma_{s}(\cdot)$. Suppose that

H1) the system $\dot{\xi}=f(\xi)+g(\xi) u$ is ISS with respect to the input $u$, and $\dot{\xi}=f(\xi)$ is LES;

H2) $H F^{-1} G<0$.

Then there exist $\kappa^{\star}>0$ and $\epsilon^{\star}>0$, such that for any $\kappa \in$ $\left(0, \kappa^{\star}\right)$ and $\epsilon \in\left(0, \epsilon^{\star}\right)$ the closed-loop system

$$
\begin{aligned}
& \dot{z}=h(\xi) \\
& \dot{\xi}=f(\xi)-g(\xi) \epsilon \sigma_{s}\left(\frac{\kappa}{\epsilon} z\right)
\end{aligned}
$$

is globally asymptotically stable (GAS)-LES.

Proof: Consider, first, the linear approximation of (3) which is given by (4). Next, rewrite the control law as

$$
\begin{aligned}
u & =-\epsilon \sigma_{s}\left(\frac{\kappa z}{\epsilon}\right)=-\kappa z-\left(\epsilon \sigma_{s}\left(\frac{\kappa z}{\epsilon}\right)-\kappa z\right) \\
& =-\kappa z-\psi(\kappa z)
\end{aligned}
$$

where $\psi(\kappa z)$ denotes a nonlinearity acting on $\kappa z$ that belongs to the sector $[-1,0]$. Consider now the system

$$
\left[\begin{array}{c}
\dot{z} \\
\dot{\xi}
\end{array}\right]=\left[\begin{array}{cc}
0 & H \\
-\kappa G & F
\end{array}\right]\left[\begin{array}{l}
z \\
\xi
\end{array}\right]-\left[\begin{array}{l}
0 \\
G
\end{array}\right] \psi(\kappa z)
$$

and note that, for sufficiently small $\kappa$ the poles of

$$
\begin{aligned}
\mathcal{G}_{n}(s) & =\left[\begin{array}{ll}
\kappa & 0
\end{array}\right]\left(s I-\left[\begin{array}{cc}
0 & H \\
-\kappa G & F
\end{array}\right]\right)^{-1}\left[\begin{array}{l}
0 \\
G
\end{array}\right] \\
& =\left(\frac{\left.\kappa G(s I-F)^{-1} H\right)}{s}\right)\left(1+\frac{\left.\kappa G(s I-F)^{-1} H\right)}{s}\right)^{-1}
\end{aligned}
$$

lie in the left-half of the complex plane, and that

$$
\left|\frac{\kappa G(j \omega I-F)^{-1} H}{j \omega}\right| \leq\left|1+\frac{\kappa G(j \omega I-F)^{-1} H}{j \omega}\right| .
$$

Hence, the system

$$
\begin{aligned}
{\left[\begin{array}{c}
\dot{z} \\
\dot{\xi}
\end{array}\right] } & =\left[\begin{array}{cc}
0 & H \\
-\kappa G & F
\end{array}\right]\left[\begin{array}{l}
z \\
\xi
\end{array}\right]-\left[\begin{array}{l}
0 \\
G
\end{array}\right] w \\
\eta & =\kappa z
\end{aligned}
$$

with input $w$ and output $\eta$ is asymptotically (exponentially) stable and has an $L_{2}$-gain not larger than one. Therefore, by the circle criterion (or the small gain theorem) we conclude GAS-LES of (6).

Consider now (5) and note that, by LES and the ISS property of the $\xi$-subsystem, if $\epsilon$ is sufficiently small, ${ }^{3}$ there exists a finite

${ }^{3}$ Recall that $\left|\sigma_{s}(\cdot)\right| \leq 1$. time $t_{o}>0$, such that for all $t \geq t_{o},\|\xi(t)\| \leq c_{1} \epsilon$, for some positive number $c_{1}$. Rewrite now (5) as

$$
\left[\begin{array}{c}
\dot{z} \\
\dot{\xi}
\end{array}\right]=\left[\begin{array}{cc}
0 & H+\delta_{H}(\xi) \\
-\kappa G & F+\delta_{F}(\xi, u)
\end{array}\right]\left[\begin{array}{c}
z \\
\xi
\end{array}\right]-\left[\begin{array}{c}
0 \\
G
\end{array}\right] \psi(\kappa z)
$$

and note that, there exist positive constants $c_{H}$ and $c_{F}$, such that for all $t \geq t_{o}$

$$
\begin{aligned}
\left\|\delta_{H}(\xi(t))\right\| & \leq c_{H} \epsilon \\
\left\|\delta_{F}(\xi(t), u(t))\right\| & \leq c_{F} \epsilon .
\end{aligned}
$$

System (9) can be regarded as a perturbed linear system with perturbations $\delta_{H}(\cdot)$ and $\delta_{F}(\cdot)$ that can be rendered asymptotically arbitrarily small reducing $\epsilon$. Note also that the perturbations are such that, if $\epsilon$ is sufficiently small, all but one of the eigenvalues of the family of systems (9) with $\kappa=0$ are in the left part of the complex plane, with the remaining eigenvalue at the origin. We conclude that there exists $\epsilon^{\star}>0$ such that for all $\epsilon \in\left(0, \epsilon^{\star}\right)$, and for all $\kappa$ sufficiently small, every element in the family of transfer functions

$$
\mathcal{G}(s)=\left[\begin{array}{ll}
\kappa & 0
\end{array}\right]\left(s I-\left[\begin{array}{cc}
0 & H+\Delta_{H} \\
-\kappa G & F+\Delta_{F}
\end{array}\right]\right)^{-1}\left[\begin{array}{c}
0 \\
G
\end{array}\right]
$$

with $\left\|\Delta_{H}\right\| \leq c_{H} \epsilon$ and $\left\|\Delta_{F}\right\| \leq c_{F} \epsilon$ has $L_{2}$-gain not larger than one. As a result, by the small gain theorem (or the circle criterion), (5) is GAS-LES.

Remark 1: Note that, if the pair $\{F, G\}$ is controllable, (3) is controllable if and only if $H F^{-1} G \neq 0$. Moreover, Hypothesis H2) is not restrictive. In fact, if $H^{-1} G>0$ the result of Proposition 1 holds with $\kappa^{\star}<0$.

We remark that $\epsilon$ is the level of saturation, whereas $\kappa$ is the feedback gain, or in other words, $\kappa z$ is the appropriate output that needs to be fed back. An interesting extension of Proposition 1 would be the iterative application of the methodology proposed. Indeed this is possible, as it will be discussed in the following sections, where, it will be proven that the closed-loop system (5) is also ISS with restrictions with respect to a new external input.

The result of Proposition 1 can be interpreted as a consequence of the circle criterion, hence this facilitates the handling of a series of system uncertainties, such as time delays. While it is known that passivity-based designs may be inadequate in the presence of delays, the result in Proposition 1 is robust against (constant) time delays in the input or output path, as summarized in the following corollary.

Corollary 1: Consider (3) and a positive constant $\tau$. Under the assumptions of Proposition 1, there exists a positive $\kappa_{1}^{\star}=$ $\kappa_{1}^{\star}(\tau)$ and an $\epsilon^{\star}>0$ such that for all $\kappa_{1} \in\left(0, \kappa_{1}^{\star}\right)$ and $\epsilon \epsilon$ $\left(0, \epsilon^{\star}\right)$ the control law

$$
u=-\epsilon \sigma_{s}\left(\frac{\kappa_{1}}{\epsilon} z(t-\tau)\right)
$$

globally asymptotically (locally exponentially) stabilizes (3). 
Proof: Note that, as before, $\left|\sigma_{s}\left(\left(\kappa_{1}\right) /(\epsilon) z(t-\tau)\right)\right| \leq 1$ for any positive constant $\tau$, thus if $\epsilon$ is small enough, $\xi$ will eventually be such that $\|\xi\|<\delta_{\epsilon}$ for some small enough constant $\delta_{\epsilon}>0$. In this slice of the state-space, we consider the system

$$
\begin{aligned}
& \dot{z}(t)=H \xi(t) \\
& \dot{\xi}(t)=F \xi(t)-G \epsilon \sigma_{s}\left(\frac{\kappa_{1}}{\epsilon} z(t-\tau)\right) .
\end{aligned}
$$

If $\mathcal{G}_{\xi}(s)$ is the transfer function of the open-loop $\xi$-subsystem with output $H \xi$, then the transfer function of the system

$$
\begin{aligned}
\dot{z}(t) & =H \xi(t) \\
\dot{\xi}(t) & =F \xi(t)+G u \\
\eta & =z(t-\tau)
\end{aligned}
$$

is $\left(e^{-s \tau} \mathcal{G}_{\xi}(s)\right) /(s)$. Note now that the Nyquist diagram of $\left(\mathcal{G}_{\xi}(s)\right) /(s)$ is bounded from the left by a vertical line, say through the point $(-(1 / \kappa), 0)$. Then, there exists a positive number $\kappa_{1}<\kappa$ such that the Nyquist diagram of $\left(e^{-s \tau} \mathcal{G}_{\xi}(s)\right) /(s)$ is also bounded from the left by a vertical line through the point $\left(-\left(1 / \kappa_{1}\right), 0\right)$. To see this, note that the term $e^{-j \omega \tau}$ does not modify the amplitude of $\left(\mathcal{G}_{\xi}(j \omega) / j \omega\right)$ and does not introduce any phase shift for $\omega \rightarrow 0$. The conclusion, therefore, follows as an application of the circle criterion. $\triangleleft$

Remark 2: Corollary 1 provides a "delay dependent" stability result, i.e., the closed-loop system is not asymptotically stable for any $\tau$, but only for $0 \leq \tau \leq \tau^{\star}$. However, unlike other delay dependent criteria, the result in Corollary 1 is constructive, i.e., for any delay $\tau$ an appropriate stabilizing feedback (10) can be found.

We are now ready to state formally the stabilization problems dealt with in this paper.

Partial state feedback stabilization problem: Consider a system described by equations of the form

$$
\begin{aligned}
\dot{z} & =J z+h(\xi)+r(\xi) u \\
\dot{\xi} & =f(\xi)+g(\xi) u
\end{aligned}
$$

where $z \in \mathbb{R}^{p}, \xi \in \mathbb{R}^{n}$, and $u \in \mathbb{R}$ and suppose the following.

A1) The system $\dot{\xi}=f(\xi)+g(\xi) u$ is ISS with respect to $u$, and $\dot{\xi}=f(\xi)$ is LES.

A2) $J+J^{\prime} \leq 0 .{ }^{4}$

Find (if possible) a positive constant $\epsilon$ and an output

$$
\eta=K z
$$

such that (12) in closed loop with the control law

$$
u=-\epsilon \sigma\left(\frac{1}{\epsilon} \eta\right)+v
$$

is LES and ISS with restrictions with respect to $v$.

Regarding this problem we define the following matrices:

$$
\begin{gathered}
\left.\left.H \triangleq \frac{\partial h(\xi)}{\partial \xi}\right|_{\xi=0} \in \mathbb{R}^{p \times n} \quad F \triangleq \frac{\partial f(\xi)}{\partial \xi}\right|_{\xi=0} \in \mathbb{R}^{n \times n} \\
R \triangleq r(0) \in \mathbb{R}^{p \times 1} \quad G \triangleq g(0) \in \mathbb{R}^{n \times 1} \\
A \triangleq\left[\begin{array}{cc}
J & H \\
0 & F
\end{array}\right] \quad B \triangleq\left[\begin{array}{l}
R \\
G
\end{array}\right] \quad C \triangleq\left[\begin{array}{ll}
I & 0
\end{array}\right]
\end{gathered}
$$

${ }^{4}$ Assumption A2) can be replaced by $J^{\prime} S+S J \leq 0$ for some $S=S^{\prime}>0$. and the approximation of (12) for small $\xi$, given by

$$
\left[\begin{array}{c}
\dot{z} \\
\dot{\xi}
\end{array}\right]=A\left[\begin{array}{l}
z \\
\xi
\end{array}\right]+B u
$$

Remark 3: Note that, as proved in [30], if the subsystem $\dot{\xi}=f(\xi)$ is GAS-LES, Assumption A1) is without loss of generality because the control can always be rescaled appropriately, provided that the whole state is measurable. However, if this rescaling is undesired, ${ }^{5}$ the minimal assumption under which the partial state feedback stabilization problem above is solvable, in the context of this work, is that the system is ISS with some restriction [18]. For example, the system $\dot{x}=-x^{3}+\left(1+x^{3}\right) u$ is not ISS, but it is ISS with the restriction $|u|<1$.

The second problem that will be solved in the paper is the problem of asymptotic stabilization of a linear stable SISO system $\dot{z}=J z+\bar{H} w$ when the available output is subject to saturation. This is formally stated as follows.

Bounded output stabilization problem: Consider a nonlinearity $\sigma(\cdot)$ and a system described by equations of the form

$$
\begin{aligned}
& \dot{z}=J z+\bar{H} w \\
& y=\sigma(K z)
\end{aligned}
$$

with $z \in \mathbb{R}^{p}, w \in \mathbb{R}$, and $y \in \mathbb{R}$. Suppose A2) holds. Find (if possible) a dynamic control law

$$
\begin{aligned}
\dot{\xi} & =F \xi-G y \\
w & =\Gamma \xi
\end{aligned}
$$

such that the closed-loop system (18)-(19) is GAS-LES.

\section{TWo USEFUL LEMMAS}

In this section, we present two lemmas that are instrumental to prove the main results of the paper. They are both related to the existence of solutions for a special matrix inequality. Note that the proofs of both these lemmas are constructive, i.e., we provide a family of solutions of the considered matrix inequality.

Lemma 1: Let $A, B$, and $C$ be defined as in (16) and suppose $\{A, B\}$ is controllable, $F \in \mathbb{R}^{n \times n}$ is a Hurwitz matrix and $J \in \mathbb{R}^{p \times p}$ is such that A2) holds. Then there exist $P \in$ $\mathbb{R}^{(n+p) \times(n+p)}$ and $K \in \mathbb{R}^{1 \times p}$ such that

$$
\begin{aligned}
\left(K C-B^{\prime} P\right)^{\prime}\left(K C-B^{\prime} P\right)+P A+A^{\prime} P & \leq 0 \\
P & =P^{\prime}>0
\end{aligned}
$$

and $A_{\mathrm{cl}}=A-B K C$ is Hurwitz.

Proof: Let $P$ be defined as

$$
P \triangleq\left[\begin{array}{cc}
\chi I & Y \\
Y^{\prime} & P_{\xi}
\end{array}\right]
$$

with $\chi$ a positive constant and $P_{\xi}=P_{\xi}^{\prime}>0$ to be selected. As a result, (20) rewrites

$$
\begin{aligned}
& {\left[\begin{array}{cc}
\chi\left(J+J^{\prime}\right) & \chi H+Y F+J^{\prime} Y \\
\chi H^{\prime}+F^{\prime} Y^{\prime}+Y^{\prime} J & Y^{\prime} H+H^{\prime} Y+P_{\xi} F+F^{\prime} P_{\xi}
\end{array}\right]} \\
& +\left[\begin{array}{c}
K^{\prime}-\chi R-Y G \\
-Y^{\prime} R-P_{\xi} G
\end{array}\right]\left[\begin{array}{c}
K^{\prime}-\chi R-Y G \\
-Y^{\prime} R-P_{\xi} G
\end{array}\right]^{\prime} \leq 0 .
\end{aligned}
$$

${ }^{5}$ This is the case when not the whole state is measurable, or when the requirements on the control signal amplitude cannot be fulfilled when a feedback transformation is applied. 
Setting

$$
K=\chi R^{\prime}+G^{\prime} Y^{\prime}
$$

the problem is translated into finding matrices $Y$ and $P_{\xi}$ such that (22) holds. To this end, note that $Y$ can always be selected such that

$$
\chi H+Y F+J^{\prime} Y=0
$$

for all $H, F, J$ and all positive constants $\chi$. With $K$ and $Y$ defined by (23) and (24), (22) reduces to

$$
\left[\begin{array}{cc}
\chi\left(J+J^{\prime}\right) & 0 \\
0 & T+P_{\xi} F+F^{\prime} P_{\xi}+P_{\xi} G G^{\prime} P_{\xi}
\end{array}\right] \leq 0
$$

where

$$
\begin{aligned}
T & =Y^{\prime} H+H^{\prime} Y+Y^{\prime} R R^{\prime} Y+Y^{\prime} R G^{\prime} P_{\xi}+P_{\xi} G R^{\prime} Y \\
& =Y^{\prime}\left(H+R G^{\prime} P_{\xi}\right)+\left(H^{\prime}+P_{\xi} G R^{\prime}\right) Y+Y^{\prime} R R^{\prime} Y
\end{aligned}
$$

Since $\chi\left(J+J^{\prime}\right) \leq 0$, the problem is reduced to finding a $P_{\xi}$ such that

$$
T+P_{\xi} F+F^{\prime} P_{\xi}+P_{\xi} G G^{\prime} P_{\xi} \leq 0 .
$$

To solve this problem, let $\bar{P}_{\xi}$ be such that $\bar{P}_{\xi} F^{\prime}+F \bar{P}_{\xi}=$ $-\left(G G^{\prime}+I\right)$. Then, setting $P_{\xi}=\bar{P}_{\xi}^{-1}$ yields

$$
P_{\xi} F+F^{\prime} P_{\xi}+P_{\xi} G G^{\prime} P_{\xi}=-P_{\xi} P_{\xi}<0 .
$$

Hence, it is sufficient to show that $T$ can be made arbitrarily small. To this end, notice that the solution of (24) is

$$
Y=\chi \bar{Y}
$$

where $\bar{Y}$ is the solution of

$$
H+\bar{Y} F+J^{\prime} \bar{Y}=0
$$

Therefore

$T=\chi\left(\bar{Y}^{\prime}\left(H+R G^{\prime} P_{\xi}\right)+\left(H^{\prime}+P_{\xi} G R^{\prime}\right) \bar{Y}\right)+\chi^{2}\left(\bar{Y}^{\prime} R R^{\prime} \bar{Y}\right)$

and this can be made arbitrarily small by a proper selection of $\chi>0$.

Besides, $P$ constructed as before can be rendered positive definite. For, note that following standard decomposition arguments $P$ is positive definite if and only if $P_{\xi}-\chi \bar{Y}^{\prime} \bar{Y}$ is positive-definite, which is true for a positive-definite $P_{\xi}$ and small enough $\chi$. Therefore, there exists a positive $\chi$ such that (20) holds.

To complete the proof we need to show that $A_{\mathrm{cl}}=A-B K C$ is Hurwitz. To this end observe that (20) is equivalent to

$$
A_{\mathrm{cl}}^{\prime} P+P A_{\mathrm{cl}}+P B B^{\prime} P+C^{\prime} K^{\prime} K C \leq 0
$$

which yields

$$
P^{-1} A_{\mathrm{cl}}^{\prime}+A_{\mathrm{cl}} P^{-1} \leq-B B^{\prime} \leq 0 .
$$

On the other hand, it is trivial to check that if $\{A, B\}$ is controllable $\left\{B^{\prime}, A_{\mathrm{cl}}\right\}$ is observable. From that and from (30), according to [39, Lemma 12.2], it is concluded that $A_{\mathrm{cl}}$ is Hurwitz.
Remark 4: Inequality (29) arises in the nonstandard $\mathcal{H}_{\infty}$ control problem [8] described by the equations

$$
\begin{aligned}
\dot{x} & =A x+B u+B w \\
z & =u \\
y & =C x
\end{aligned}
$$

where $A, B$, and $C$ are as in (16), $u$ is the control input, $w$ is the exogenous input, $z$ is the penalty variable, and $y$ is the measurement. Lemma 1 expresses the fact that there exists a static output feedback control law $u=-K y$ rendering (31) asymptotically stable and with a $L_{2}$-gain from $w$ to $z$ less than or equal to one. Note that if $J$ has eigenvalues on the $j \omega$ axis then $\gamma=1$ is the smallest achievable $L_{2}$-gain for (31), i.e., any static or dynamic output feedback stabilizing controller yields a closed-loop system with $L_{2}$-gain larger or equal to one.

Lemma 2: Let $J \in \mathbb{R}^{p \times p}, \bar{H} \in \mathbb{R}^{p \times 1}$, and $K \in \mathbb{R}^{1 \times p}$ be known matrices such that A2) holds, $\left\{J^{\prime}, \bar{H}\right\}$ is controllable and $\{K, J\}$ is observable. Then there exist $P \in \mathbb{R}^{(2 p) \times(2 p)}, G \in$ $\mathbb{R}^{p \times 1}, \Gamma \in \mathbb{R}^{1 \times p}$ and a Hurwitz matrix $F \in \mathbb{R}^{p \times p}$ such that (20) holds, with $R=0, H=\bar{H} \Gamma$, and $A, B$, and $C$ as in (16), and the matrix $A_{\mathrm{cl}}=A-B K C$ is Hurwitz.

Proof: Partition $P$ as in (21) and repeat the first steps of the Proof of Lemma 1. However, note that we are looking now for $F, G$, and $\Gamma$. Let $F$ be a Hurwitz matrix with distinct eigenvalues, and $L$ be such that $\operatorname{spec}\left(J^{\prime}+\bar{H} L\right)=\operatorname{spec}(-F)$. Note that such an $L$ exists because of controllability of the pair $\left\{J^{\prime}, \bar{H}\right\}$. Then there exists a nonsingular matrix $X$ such that

$$
J^{\prime} X+\bar{H} L X+X F=0 .
$$

Therefore, setting $Y=\chi X$, for some positive $\chi$, solves the Sylvester equation (24) with $H=\bar{H} L X$. Next, set $\Gamma=L X, G=Y^{-1} K^{\prime}$ and let $\bar{P}_{\xi}$ be the positive-definite matrix that solves the Lyapunov equation

$$
\bar{P}_{\xi} F^{\prime}+F \bar{P}_{\xi}=-\left(X^{-1} K^{\prime} K\left(X^{-1}\right)^{\prime}+I\right) .
$$

Choosing $P_{\xi}=\chi^{2} \bar{P}_{\xi}^{-1}$, it is easy to verify that the first of inequalities (20) holds for a large enough $\chi>0$. On the other hand, with the above selections for $P_{\xi}$ and $Y$, the matrix $P$ is positive definite for a large enough $\chi>0$.

Observe, now, that (20), or the equivalent inequality (29), yields

$A_{\mathrm{cl}}^{\prime} P+P A_{\mathrm{cl}} \leq-\left(P B B^{\prime} P+C^{\prime} K^{\prime} K C\right) \leq-C^{\prime} K^{\prime} K C \leq 0$

and that observability of the pair $\{K, J\}$ implies detectability of the pair $\left\{K C, A_{\mathrm{cl}}\right\}$. As a result, by [39, Lemma 12.2], $A_{\mathrm{cl}}$ is Hurwitz.

Remark 5: Dual to what stated in Remark 4, consider the nonstandard $\mathcal{H}_{\infty}$ control problem described by the equations

$$
\begin{aligned}
\dot{x} & =J x+\bar{H} u \\
z & =K x \\
y & =K x+w
\end{aligned}
$$

where $J$ is such that A2) holds, $u$ is the control input, $w$ is the exogenous input, $z$ is the penalty variable, and $y$ is the measurement. Lemma 2 expresses the fact that there exists a dynamic 
output feedback control law, of the same dimension as system (33), described by equations of the form

$$
\begin{aligned}
u & =\Gamma \xi \\
\dot{\xi} & =F \xi-G y
\end{aligned}
$$

such that the closed-loop system (33)-(34) is asymptotically stable and with an $L_{2}$-gain from $w$ to $z$ less than or equal to one. Note that if $J$ has eigenvalues on the $j \omega$ axis then an $L_{2}$-gain equal to one is the smallest achievable gain, with any output feedback.

Remark 6: The results in Lemmas 1 and 2 can be trivially given a multivariable control extension. Namely, under the assumptions of Lemma 1, for $G \in \mathbb{R}^{n \times m}$ and $R \in \mathbb{R}^{p \times m}$ there exist a matrix $K \in \mathbb{R}^{m \times p}$ and a positive-definite matrix $P \in$ $\mathbb{R}^{(n+p) \times(n+p)}$ such that (20) holds and $A_{\mathrm{cl}}=A-B K C$ is Hurwitz. Similarly, under the assumptions of Lemma 2, for $\bar{H} \in$ $\mathbb{R}^{p \times m}$ and $K \in \mathbb{R}^{q \times p}$ (system with $m$ inputs and $q$ outputs) there exist matrices $G \in \mathbb{R}^{p \times q}, \Gamma \in \mathbb{R}^{m \times p}$ and a positive-definite $P \in \mathbb{R}^{2 p \times 2 p}$ such that (20) holds.

\section{Stabilization With Bounded Partial State Feedback}

In this section, we provide our main result on the stabilization, with partial state feedback bounded control, of systems described by (12).

Proposition 2: Consider a nonlinearity $\sigma(\cdot)$ belonging to the sector $[0,1]$ and the system described by the equations

$$
\begin{aligned}
\dot{z} & =J z+h(\xi)+r(\xi) u \\
\dot{\xi} & =f(\xi)+g(\xi) u
\end{aligned}
$$

with $z \in \mathbb{R}^{p}, \xi \in \mathbb{R}^{n}, u \in \mathbb{R}$, and $f(0)=0, h(0)=0$. Suppose A1) and A2) hold and, moreover, assume the following.

C1) The linear approximation of (35) is controllable.

Then there exists $\epsilon^{\star}>0$ and a matrix $K \in \mathbb{R}^{1 \times p}$ such that if $\epsilon \in\left(0, \epsilon^{\star}\right)$, the static partial state feedback control law

$$
u=-\epsilon \sigma\left(\frac{1}{\epsilon} K z\right)
$$

globally stabilizes (35). Moreover, if

C2) all trajectories $z(t)$ of $\dot{z}(t)=J z(t)$ such that $\sigma(K z(t))=0$, for all $t \geq 0$, are such that $\lim _{t \rightarrow \infty} z(t)=0$.

Then (36) globally asymptotically (locally exponentially) stabilizes (35). Furthermore, the system

$$
\begin{aligned}
& \dot{z}=J z+h(\xi)-r(\xi) \epsilon \sigma_{s}\left(\frac{1}{\epsilon} K z\right)+r(\xi) w \\
& \dot{\xi}=f(\xi)-g(\xi) \epsilon \sigma_{s}\left(\frac{1}{\epsilon} K z\right)+g(\xi) w
\end{aligned}
$$

is ISS with respect to the new input $w$, with the restriction $|w| \leq$ $\mu$, with $\mu<\epsilon$.

Remark 7: The second claim of Proposition 2 holds with the choice of the "nonlinearity" $\sigma(s)=0$, for all $s \in \mathbb{R}$. This is due to Assumption C2), which, in this case, implies that system
(35) is the interconnection of two asymptotically stable systems, possessing bounded trajectories and operating in open loop.

Remark 8: A similar result has been proven in [10, Th. 14.3.3] on the basis of the results in [36]. Note, however that the result of [10, Th. 14.3.3] requires, in general, full state feedback, and that the result in Proposition 2 is based on a different construction. As a result, Proposition 2 can also be used in the design of output feedback control laws (see Section V), in the design of quantized or constant sign controllers (see Corollaries 2 and 3 ) and when dealing with some robustness problems (see Corollary 1). In fact, the assumptions on the system in [10, Th. 14.3.3] are different to the assumptions in Proposition 2. Therein, the construction uses the fact that the pair $\{J, R\}$ is stabilizable, while a cross-term corresponding to $h(\xi)$ of (35) is assumed to be of order at least two. Under this assumptions the feedback used in [10, Th. 14.3.3] is of the form (36), but this time $K$ is such that $J-R K$ is Hurwitz. From what will become clear from the Proof of Proposition 2 and the examples presented in the rest of the paper, it is obvious that the two results are not addressing the same problem. For example, Proposition 2 also deals with the case where $J$ is skew symmetric and $R=0$, i.e., the pair $\{J, R\}$ is not stabilizable, and the upper subsystem is driven entirely by $\xi$.

Proof: As discussed earlier, because of A1), there exists $\epsilon^{\star}>0$ such that if $\epsilon \in\left(0, \epsilon^{\star}\right)$, the state of the closed loop system (35)-(36) will in finite time enter a small enough "slice" where $\|\xi\|<\delta_{\epsilon}$, for an arbitrarily small $\delta_{\epsilon}>0$. There, we can consider the approximation of (35) for small $\|\xi\|$, as explained in the proof of Proposition 1. In other words, it suffices to study the stabilization with bounded control problem for (17) to obtain stabilization results for the nonlinear system (12).

Denoting $x=\left[\begin{array}{ll}z^{\prime} & \xi^{\prime}\end{array}\right]^{\prime}$, the state space equations of the cascade (12) and the output described in the partial-state feedback stabilization problem (13) are written as

$$
\begin{aligned}
\dot{x} & =A x+B u \\
\eta & =K C x .
\end{aligned}
$$

Let $K$ be a matrix such that the linear feedback $u_{l}=-K C x$ exponentially stabilizes system (17). The proposed control law (36) can be written as

$$
\begin{aligned}
u & =-\epsilon \sigma\left(\frac{1}{\epsilon} \eta\right)=-\left[K C x+\left(\epsilon \sigma\left(\frac{1}{\epsilon} \eta\right)-K C x\right)\right] \\
& =-K C x-\psi(\eta)
\end{aligned}
$$

where $\psi(\eta)$ is a new nonlinearity restricted to the sector $[-1,0]$. Note that up to now, $K$ is some matrix that sets $A_{\mathrm{cl}}$ to be Hurwitz. However, to prove stability in the presence of the nonlinearity $\psi(\eta)$ a special "stabilizing" $K$ has to be selected. For, note that (36)-(38) can be regarded as the feedback interconnection of the system

$$
\begin{aligned}
& \dot{x}=A_{\mathrm{cl}} x+B v \\
& \eta=K C x
\end{aligned}
$$

where $A_{\mathrm{cl}} \triangleq A-B K C$, with $v=-\psi(\eta)$. Moreover, the $L_{2}$-gain of $\psi(\eta)$ is not larger than one, hence, selecting $K$ sat- 
isfying inequality (20) for some $P>0$, yields, ${ }^{6}$ by Assumption $\mathrm{C} 2$ ) and the generalized small gain theorem in [26], an asymptotically stable closed-loop system. Moreover, $A_{\mathrm{cl}}$ is a Hurwitz matrix, from Lemma 1.

To complete the Proof of Proposition 2, we need to prove the ISS property of (37). ${ }^{7}$ First notice, that if $\epsilon \in\left(0, \epsilon^{\star}\right)$, for any $w$ such that $|w|<\epsilon$, in finite time, all trajectories of the nonlinear system (37) will eventually be such that $\|\xi(t)\|<\delta_{\epsilon}^{\prime}$ for all $t \geq \bar{t}$. Therein, we consider the approximation of system (37) for small $\|\xi\|$

$$
\dot{x}=A x-B \epsilon \sigma_{s}\left(\frac{\eta}{\epsilon}\right)+B w
$$

and we prove that it is ISS with some restriction on $w$. For, consider the positive-definite function $V=x^{\prime} P x$, with $P$ as defined in (21). Along the trajectories of (40), one has

$$
\dot{V}=x^{\prime}\left(A^{\prime} P+P A\right) x-2 x^{\prime} P B\left(\epsilon \sigma_{s}\left(\frac{\eta}{\epsilon}\right)-w\right)
$$

where $\eta=K z$. With simple calculations, using (24) and (27), it is easy to see that

$$
\begin{aligned}
x^{\prime}\left(A^{\prime} P+P A\right) x=\chi z^{\prime}(J & \left.+J^{\prime}\right) z \\
& -\xi^{\prime}\left(Q_{\xi}-\chi\left(H^{\prime} \bar{Y}+\bar{Y}^{\prime} H\right)\right) \xi
\end{aligned}
$$

where $Q_{\xi}$ is a positive-definite matrix and $P_{\xi}$ is the positive-definite solution of the Lyapunov equation $F^{\prime} P_{\xi}+P_{\xi} F=$ $-Q_{\xi}$. Note that $P_{\xi}$ and $Q_{\xi}$ are as in the proof of Lemma 1. Note also that, by Assumption A2), $J+J^{\prime}$ is negative-semidefinite and that

$$
P B=\left[\begin{array}{c}
\chi R+Y G \\
Y^{\prime} R+P_{\xi} G
\end{array}\right]=\left[\begin{array}{c}
K^{\prime} \\
Y^{\prime} R+P_{\xi} G
\end{array}\right] .
$$

As a result, by simple manipulations, (41) becomes

$$
\begin{aligned}
\dot{V}= & \chi z^{\prime}\left(J+J^{\prime}\right) z-\xi^{\prime}\left(Q_{\xi}-\chi\left(H^{\prime} \bar{Y}+\bar{Y}^{\prime} H\right)\right) \xi \\
& -2 \xi^{\prime}\left(Y^{\prime} R+P_{\xi} G\right)\left(\epsilon \sigma_{s}\left(\frac{\eta}{\epsilon}\right)-w\right) \\
& -2 \eta\left(\epsilon \sigma_{s}\left(\frac{\eta}{\epsilon}\right)-w\right)+\left[\epsilon \sigma_{s}\left(\frac{\eta}{\epsilon}\right)-w\right]^{2} \\
& -\left[\epsilon \sigma_{s}\left(\frac{\eta}{\epsilon}\right)-w\right]^{2}
\end{aligned}
$$

hence

$$
\begin{aligned}
\dot{V} \leq & -\left[\epsilon \sigma_{s}\left(\frac{1}{\epsilon} \eta\right)-w, \xi^{\prime}\right] \\
& \times\left[\begin{array}{cc}
1 & \chi R^{\prime} \bar{Y}+G^{\prime} P_{\xi} \\
\chi \bar{Y}^{\prime} R+P_{\xi} G & Q_{\xi}-\chi\left(H^{\prime} \bar{Y}+\bar{Y}^{\prime} H\right)
\end{array}\right] \\
& \times\left[\begin{array}{c}
\epsilon \sigma_{s}\left(\frac{1}{\epsilon} \eta\right)-w \\
\xi
\end{array}\right] \\
& -\left[\epsilon \sigma_{s}\left(\frac{1}{\epsilon} \eta\right)-w\right]\left(2 \eta-\left[\epsilon \sigma_{s}\left(\frac{1}{\epsilon} \eta\right)-w\right]\right) .
\end{aligned}
$$

The matrix

$$
M=\left[\begin{array}{cc}
1 & \chi R^{\prime} \bar{Y}+G^{\prime} P_{\xi} \\
\chi \bar{Y}^{\prime} R+P_{\xi} G & Q_{\xi}-\chi\left(H^{\prime} \bar{Y}+\bar{Y}^{\prime} H\right)
\end{array}\right]
$$

${ }^{6}$ Recall that, by Remark 4 and Lemma 1 , system (39) has a $L_{2}$-gain $\left(\mathcal{H}_{\infty}\right.$-norm) less than or equal to one.

${ }^{7}$ Note that the symmetric nonlinearity $\sigma_{s}(s)$ is used. is positive-definite by construction, as shown in the proof of Lemma 1.

Under the restriction $|w|<\epsilon$, we see that the following implications hold:

$$
\begin{aligned}
|\eta| & >|w| \\
& \Rightarrow\left\{\operatorname{sign}\left[\epsilon \sigma_{s}\left(\frac{1}{\epsilon} \eta\right)-w\right]=\operatorname{sign}(\eta)\right. \\
& \operatorname{sign}\left(2 \eta-\left[\epsilon \sigma_{s}\left(\frac{1}{\epsilon} \eta\right)-w\right]\right) \\
& \left.=\operatorname{sign}\left(\left(\eta-\epsilon \sigma_{s}\left(\frac{1}{\epsilon} \eta\right)\right)+(\eta+w)\right)=\operatorname{sign}(\eta)\right\} \\
& \Rightarrow-\left[\epsilon \sigma_{s}\left(\frac{1}{\epsilon} \eta\right)-w\right]\left(2 \eta-\left[\epsilon \sigma_{s}\left(\frac{1}{\epsilon} \eta\right)-w\right]\right)<0 \\
& \Rightarrow \dot{V} \leq 0 .
\end{aligned}
$$

This means that (40) with output $\eta$ is input-to-output stable with some (nonzero) restriction on $w$. Using the result in [31], to prove ISS it is sufficient to show that the pair $\{K C, A\}$ is detectable. To this end, note that the matrix $A_{\mathrm{cl}}^{\prime}=A^{\prime}-C^{\prime} K^{\prime} B^{\prime}$ is Hurwitz, therefore the pair $\left\{A^{\prime}, C^{\prime} K^{\prime}\right\}$ is stabilizable. Thus, by [39, Prop. 3.1], the pair $\{K C, A\}$ is detectable. This completes the Proof of Proposition 2.

Remark 9: In light of Remark 6, if we consider a system of the form (35) where $u \in \mathbb{R}^{m}$, then, under the assumptions of Proposition 2, there exists a matrix $K \in \mathbb{R}^{m \times p}$ with $K=$ $\left[k_{1}, k_{2}, \ldots, k_{m}\right]^{\prime}$ such that the control law

$$
u=-\left[\begin{array}{c}
\epsilon_{1} \sigma_{s}\left(\frac{1}{\epsilon_{1}} k_{1} z\right) \\
\epsilon_{2} \sigma_{s}\left(\frac{1}{\epsilon_{2}} k_{2} z\right) \\
\vdots \\
\epsilon_{m} \sigma_{s}\left(\frac{1}{\epsilon_{m}} k_{m} z\right)
\end{array}\right]
$$

globally asymptotically stabilizes the underlying system.

Remark 10: System (35) with output $\eta$ is not, in general, minimum phase, nor with relative degree one. This fact distinguishes the present stabilization method from a family of other nonlinear control results that rely on some passivity property of the system; see, for example, [10, Prop. 14.1.5] or even the results in [35] and [33].

It is easy to see that cascades with a simple integrator for the upper system (see also Proposition 1) belong to the class of systems described by (35) with $J=0$ and $r(\xi)=0$. In this case we can name the 'desired output' mentioned in the stabilization problem as $\eta=\kappa z$, where $\kappa$ is as described in Proposition 1. In general, when integrators are present, special attention has to be given to the choice of the nonlinearity $\sigma(\cdot)$. Note for example, that using the nonnegative nonlinearity $\sigma_{+}(\cdot)$ for the system (3) we cannot achieve GAS, since there are no isolated equilibria (the trajectories of the system can converge to any point $\left[z_{-}, 0\right]$, where $z_{-} \leq 0$ ). However, when $J$ is a full-rank matrix, the equilibrium is always uniquely defined, hence GAS can be achieved. On the other hand, if the aim is not to globally asymptotically stabilize (35) but to practically stabilize it, i.e., to achieve convergence to a small enough neighborhood of the origin, then the saturation function could be like $\sigma_{q}(\cdot)$ of (2). This discussion can be formally summarized as follows. 
Corollary 2: Consider system (35). Suppose conditions A1) and A2) hold. Suppose moreover that $J$ is a full-rank matrix. Then there exists $\epsilon^{\star}>0$ and a matrix $K \in \mathbb{R}^{1 \times p}$ such that if $\epsilon \in\left(0, \epsilon^{\star}\right)$, the static partial state feedback control law

$$
u=-\epsilon \sigma_{+}\left(\frac{1}{\epsilon} K z\right) \quad\left(\text { or } \quad u=\epsilon \sigma_{+}\left(-\frac{1}{\epsilon} K z\right)\right)
$$

globally asymptotically stabilizes system (35). Moreover, $u(t) \leq 0(\text { or } u(t) \geq 0)^{8}$ for all $t \geq 0$.

Proof: Note that if $J$ is a full rank matrix, then the linear approximation of system (35) is controllable as long as the $\xi$-subsystem is controllable, and the matrices $H$ and $R$ are not both zero. Also, the half space defined by

$$
\begin{aligned}
& \mathbb{S} \triangleq\left\{z \in \mathbb{R}^{p}: \sigma_{+}\left(\frac{1}{\epsilon} K z\right)=0\right\}=\left\{z \in \mathbb{R}^{p}: K z \leq 0\right\} \\
& \left(\text { or } \mathbb{S} \triangleq\left\{z \in \mathbb{R}^{p}: \sigma_{+}\left(-\frac{1}{\epsilon} K z\right)=0\right\}\right. \\
& \left.\quad=\left\{z \in \mathbb{R}^{p}: K z \geq 0\right\}\right)
\end{aligned}
$$

contains the point $z=0$ but does not contain any neighborhood of $z=0$. Therefore, the only trajectory of $\dot{z}=J z$ contained in $\mathbb{S}$ is such that $\lim _{t \rightarrow \infty} z(t)=0 .{ }^{9} \mathrm{As}$ a result, conditions $\left.\mathrm{C} 1\right)$ and C2) are satisfied, and the result follows from Proposition $2 . \triangleleft$

Corollary 3: Consider system (3). Suppose that assumptions H1) and H2) of Proposition 1 hold. Then, there exist $\kappa^{\star}>$ $0, \epsilon^{\star}>0$, and $t_{o} \in \mathbb{R}_{+}$such that for any $\kappa \in\left(0, \kappa^{\star}\right)$ and $\epsilon \in\left(0, \epsilon^{\star}\right)$ all trajectories $(z(t), \xi(t))$ of the closed-loop system

$$
\begin{aligned}
\dot{z} & =h(\xi) \\
\dot{\xi} & =f(\xi)-g(\xi) \epsilon \sigma_{q}\left(\frac{\kappa}{\epsilon} z\right)
\end{aligned}
$$

are such that

$$
\lim _{t \rightarrow \infty} \xi(t)=0 \quad \text { and } \quad|z(t)| \leq \frac{\epsilon}{\kappa} \quad \forall t \geq t_{o} .
$$

Proof: As in the Proof of Propositions 1 and 2, we focus on the approximated system for small $\|\xi\|$. For such a system, consider the Lyapunov function

$$
V=\left[z, \xi^{\prime}\right]\left[\begin{array}{cc}
\chi & Y \\
Y^{\prime} & P_{\xi}
\end{array}\right]\left[\begin{array}{c}
z \\
\xi
\end{array}\right]
$$

with $P_{\xi}=P_{\xi}^{\prime}>0$ such that ${ }^{10} F^{\prime} P_{\xi}+P_{\xi} F=-P_{\xi} G G^{\prime} P_{\xi}-$ $P_{\xi} P_{\xi}=-Q_{\xi}, Q_{\xi}=Q_{\xi}^{\prime}>0$ and $Y=-\chi H F^{-1}$. Along the trajectories of (48) one has

$$
\begin{aligned}
\dot{V}=-\xi^{\prime}\left(Q_{\xi}+\chi\right. & \left.\left(H^{\prime} H F^{-1}+F^{-T} H^{\prime} H\right)\right) \xi \\
& -2 \kappa z \epsilon \sigma_{q}\left(\frac{\kappa}{\epsilon} z\right)-2 \xi^{\prime} P_{\xi} G \epsilon \sigma_{q}\left(\frac{\kappa}{\epsilon} z\right)
\end{aligned}
$$

${ }^{8}$ Note that $\sigma_{+}(-\eta)=-\sigma_{-}(\eta)$, where $\sigma_{-}(\cdot)$ is defined in similar way to $\sigma_{+}(\cdot)$, but is equal to zero for all $\eta>0$. Like the nonlinearities (2), $\sigma_{-}(\cdot)$ also belongs to the sector $[0,1]$.

${ }^{9}$ This is due to the fact that because det $J \neq 0$, the system $\dot{z}=J z$ has no trajectory with a component of the form $z_{i}(t)=c$, with $c \neq 0$.

${ }^{10}$ See also (26) in the proof of Lemma 1. with $\kappa=-\chi H F^{-1} G>0$. Consider now the following two exclusive cases.

- $|z| \geq(\epsilon / \kappa)$. In this case $\sigma_{q}((\kappa / \epsilon) z) \neq 0$, hence, using the fact that $-y \sigma(y) \leq-[\sigma(y)]^{2}$

$$
\begin{aligned}
\dot{V} \leq & -\xi^{\prime}\left(Q_{\xi}+\chi\left(H^{\prime} H F^{-1}+F^{-T} H^{\prime} H\right)\right) \xi \\
& -2 \epsilon^{2}\left[\sigma_{q}\left(\frac{\kappa}{\epsilon} z\right)\right]^{2}-2 \xi^{\prime} P_{\xi} G \epsilon \sigma_{q}\left(\frac{\kappa}{\epsilon} z\right) \\
= & -\left[\epsilon \sigma_{q}\left(\frac{\kappa}{\epsilon} z\right), \xi^{\prime}\right] \\
& \times\left[\begin{array}{cc}
1 & G^{\prime} P_{\xi} \\
P_{\xi} G & Q_{\xi}+\chi\left(H^{\prime} H F^{-1}+F^{-T} H^{\prime} H\right)
\end{array}\right] \\
& \times\left[\begin{array}{c}
\epsilon \sigma_{q}\left(\frac{\kappa}{\epsilon} z\right) \\
\xi
\end{array}\right] \\
\triangleq & -W_{1}(x) .
\end{aligned}
$$

It is easy to see that, for a small enough $\chi, W_{1}(x)>0$.

- $|z|<(\epsilon / \kappa)$. In this case, $\sigma_{q}((\kappa / \epsilon) z)=0$ and

$$
\dot{V}=-\xi^{\prime}\left(Q_{\xi}+\chi\left(H^{\prime} H F^{-1}+F^{-T} H^{\prime} H\right)\right) \xi \triangleq-W_{2}(\xi) .
$$

Note that the matrix $Q_{\xi}+\chi\left(H^{\prime} H F^{-1}+F^{-T} H^{\prime} H\right)$ can be made positive-definite with an appropriate choice of $Q_{\xi}$ and a small enough $\chi>0$.

From the above, we can see that $\dot{V}(x)$ is bounded from above by a negative semidefinite function, namely

$$
\dot{V}(x) \leq-\min \left\{W_{1}(x), W_{2}(\xi)\right\} \leq 0 .
$$

As a result, by LaSalle's invariance principle, the trajectories of (48) are bounded and asymptotically converging to the set

$$
\begin{aligned}
\left\{z \in \mathbb{R}: \sigma_{q}\left(\frac{\kappa}{\epsilon} z\right)=0\right\} \times & \{\xi=0\} \\
& =\left\{z \in \mathbb{R}:|z|<\frac{\epsilon}{\kappa}\right\} \times\{\xi=0\} .
\end{aligned}
$$

The extension of Corollary 3 for (35) is straightforward and is omitted here for the sake of brevity, see [12].

In Figs. 1 and 2, we illustrate the conclusion of Corollaries 2 and 3 with some simulation results for a fourth order system with states $z_{1}, \ldots, z_{4}$ and control $u$. The open-loop eigenvalues are at $\pm 2 j, \pm j$. The "chattering" of the control signal observed in the top graphs of Fig. 2 can be reduced if, instead of the simple quantized nonlinearity $\sigma_{q}(\cdot)$ of (2), we use a nonlinearity with hysteresis. In the bottom graphs of Fig. 2, we show the improved simulation results, where hysteresis has been implemented.

\section{Stabilization With Sensors Saturations}

In this section, we consider the asymptotic stabilization of linear stable systems for which the measured output is subject to a constraint, for example the case where the measurement device has some range limitations. Consider a SISO linear system with saturated output, namely

$$
\begin{aligned}
\dot{z} & =J z+\bar{H} w \\
\eta & =\sigma(K z)
\end{aligned}
$$



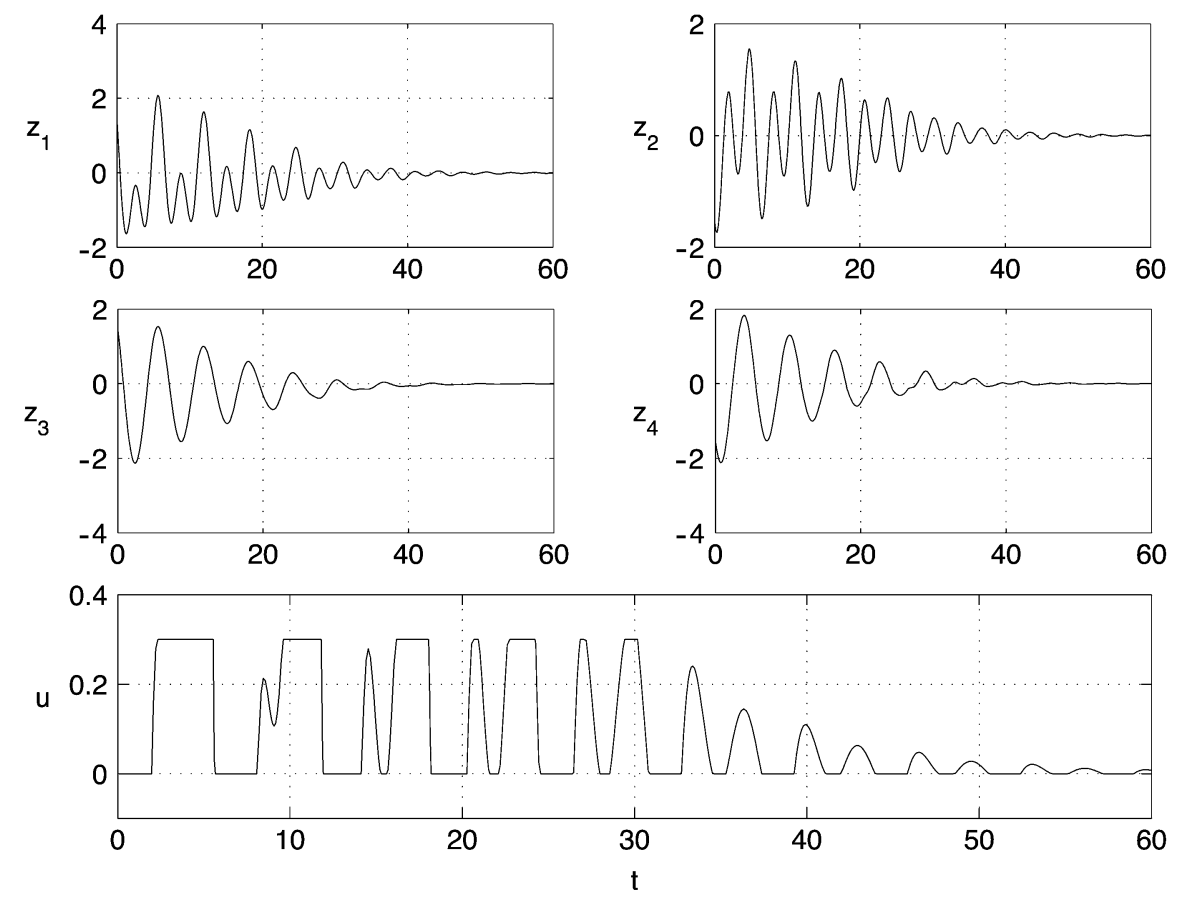

Fig. 1. Fourth-order linear system with two pairs of open-loop imaginary eigenvalues, in closed loop with a positive control law of the form (47).

with $J$ such that A2) is satisfied (i.e., $J+J^{\prime} \leq 0$ ). Goal of this section is to show that (51) is globally asymptotically stabilizable by dynamic output feedback, as illustrated in the following proposition.

Proposition 3: Consider system (51) with $z \in \mathbb{R}^{p}, w \in$ $\mathbb{R}, \eta \in \mathbb{R}$, and $J$ and $\sigma(\cdot)$ such that Assumptions A2) and C2) hold. Assume that the pair $\left\{J^{\prime}, \bar{H}\right\}$ is controllable and the pair $\{K, J\}$ is observable. Then there exist matrices $\Gamma \in \mathbb{R}^{1 \times p}, G \in$ $\mathbb{R}^{p \times 1}$ and a Hurwitz matrix $F \in \mathbb{R}^{p \times p}$ such that (51) in closed loop with the dynamic controller

$$
\begin{aligned}
\dot{\xi} & =F \xi-G \eta \\
w & =\Gamma \xi
\end{aligned}
$$

is globally asymptotically (locally exponentially) stable.

Proof: It is trivial to verify that the closed-loop system (51)-(52) is described by equations of the form

$$
\begin{aligned}
\dot{z} & =J z+\bar{H} \Gamma \xi \\
\dot{\xi} & =F \xi+G u \\
u & =-\sigma(K z)
\end{aligned}
$$

i.e., it is the feedback interconnection of a system of the form (17) with $H=\bar{H} \Gamma$ and $R=0$, and the nonlinear feedback $u=-\sigma(K z)$. Hence, selecting $\Gamma, G$ and a Hurwitz matrix $F$ as in the proof of Lemma 2 and using arguments similar to those in the proof of Proposition 2, it follows that the interconnection is globally asymptotically stable.

Remark 11: Proposition 3 can be easily extended, using the same arguments as in the proof of Corollaries 2 and 3 , to the case $\sigma(\cdot)=\sigma_{+}(\cdot)$, provided that $\operatorname{det}(J) \neq 0$, or to the case $\sigma(\cdot)=$ $\sigma_{q}(\cdot)$, if one is interested in practical, rather than asymptotic, stability.
It should be noted that the result of Proposition 3 is not restricted by the sign of the system zeros, i.e., it is applicable to both minimum and nonminimum phase systems. In the light of Remarks 6 and 9 it is also applicable to MIMO systems. Other extensions and discussions on the bounded output stabilization problem are discussed in detail in [13].

\section{APPLICATIONS}

In this section, we consider some applications of the main results of Section IV, namely the global asymptotic stabilization of a chain of integrators with bounded input, the global asymptotic stabilization of linear null controllable systems by positive (negative) control, the global asymptotic stabilization of the benchmark TORA system and the global asymptotic stabilization of underactuated ships moving on a linear course.

\section{A. Stabilization of a Chain of Integrators With Bounded Control Revisited}

The problem of global asymptotic stabilization of a chain of integrators with bounded control has been extensively studied by several researchers. In this section we revisit it, and in the light of the results of Propositions 1 and 2, we present a novel stabilizing bounded control law, complete with some remarks on its robustness.

Proposition 4: Consider the system

$$
\begin{aligned}
& \dot{x}_{1}=x_{2} \\
& \vdots \\
& \dot{x}_{n-1}=x_{n} \\
& \dot{x}_{n}=u .
\end{aligned}
$$



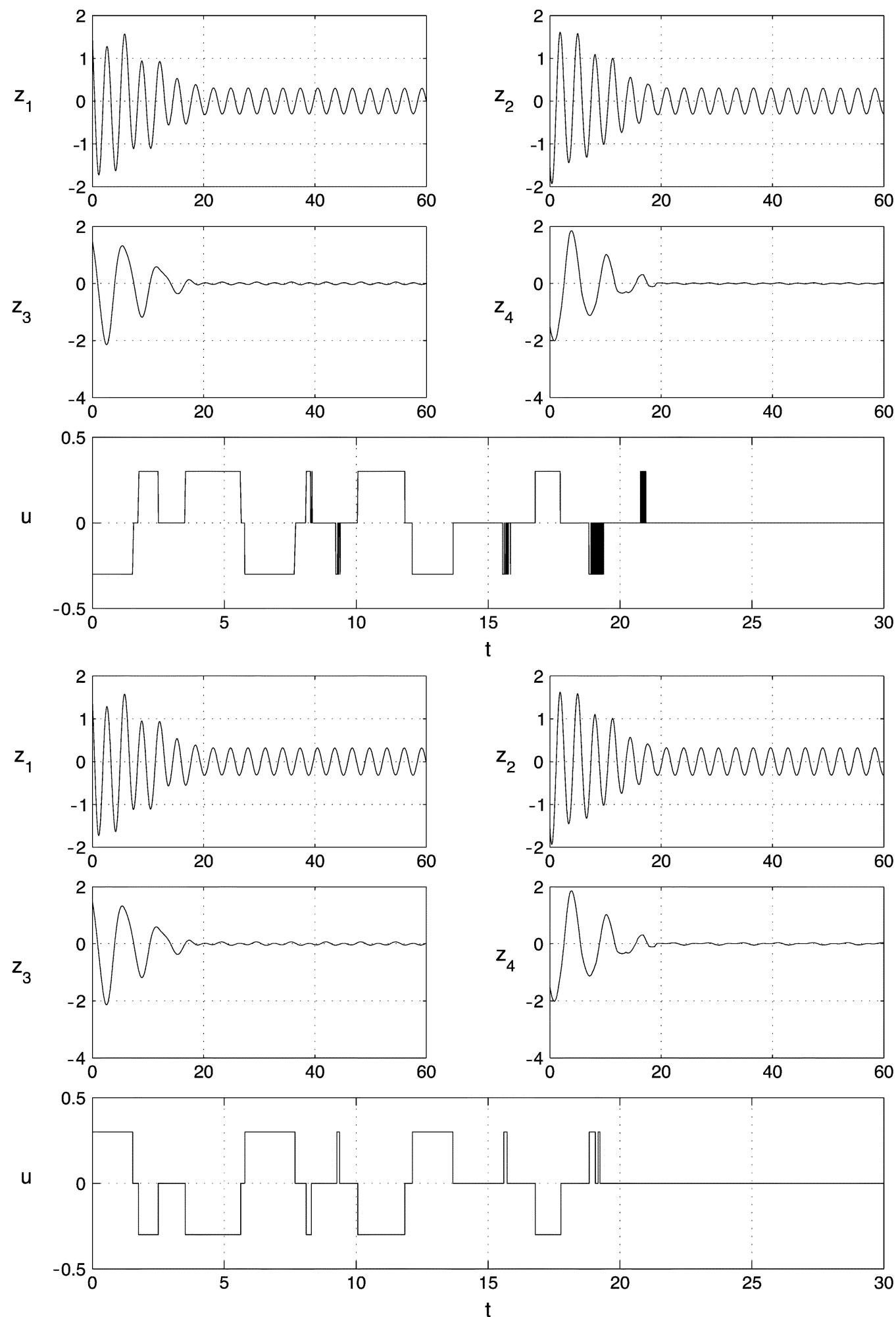

Fig. 2. Fourth-order linear system with two pairs of open-loop imaginary eigenvalues, in closed loop with a control law of the form (36) with a quantized saturation function $\sigma_{q}(s)$ (top graphs) and with a quantized control law with hysteresis (bottom graphs).

There exist positive numbers $\lambda_{1}, \lambda_{2}, \ldots, \lambda_{n-1}, \lambda_{n}$ such that, for any $\epsilon>0$, (54) in closed loop with

$$
\begin{aligned}
& u=-\frac{\epsilon}{2} \sigma_{s}\left(\frac{2 \lambda_{n}}{\epsilon} x_{n}\right)-\frac{\epsilon}{4} \sigma_{s}\left(\frac{4 \lambda_{n-1}}{\epsilon} x_{n-1}\right) \\
& -\frac{\epsilon}{8} \sigma_{s}\left(\frac{8 \lambda_{n-2}}{\epsilon} x_{n-2}\right)-\cdots-\frac{\epsilon}{2^{n}} \sigma_{s}\left(\frac{2^{n} \lambda_{1}}{\epsilon} x_{1}\right)+w
\end{aligned}
$$

is LES and ISS with the restriction $|w|<(\epsilon) /\left(2^{n+1}\right)$. Moreover, if $w=0,|u|<\epsilon$.

Proof: The proof can be carried out iteratively. For, set $u=-(\epsilon / 2) \sigma_{s}\left(\left(2 \lambda_{n}\right) /(\epsilon) x_{n}\right)+v_{n-1}$ and note that the system

$$
\begin{aligned}
\dot{x}_{n-1} & =x_{n} \\
\dot{x}_{n} & =-\frac{\epsilon}{2} \sigma_{s}\left(\frac{2 \lambda_{n}}{\epsilon} x_{n}\right)+v_{n-1}
\end{aligned}
$$


satisfies the assumptions of Proposition $1^{11}$ for every $\lambda_{n}>0$. It is also obvious that the last equation of (56) represents an ISS system with the restriction $\left|v_{n-1}\right|<(\epsilon / 2)$. As a result, there exists a positive $\lambda_{n-1}$ such that

$$
v_{n-1}=-\frac{\epsilon}{4} \sigma_{s}\left(\frac{4 \lambda_{n-1}}{\epsilon} x_{n-1}\right)+v_{n-2}
$$

achieves input to state stability of (56), with the restriction $\left|v_{n-2}\right|<(\epsilon / 4)$, and local exponential stability for $v_{n-2}=0$, according to Propositions 1 and 7 .

The proof is then completed by recursive application of Proposition 1. We remark that at each step the positive constant $\lambda_{i} \in\left(0, \lambda_{i}^{\star}\right)$ that will achieve absolute stability (see the proof of Proposition 2 or the proof of Proposition 1) will automatically belong to the set of positive $\lambda_{i}$ that would achieve exponential stability, if linear feedback was used. Also, we can see that, at each step $i$, the transfer function of the system

$$
\begin{aligned}
\dot{x}_{i} & =x_{i+1} \\
& \vdots \\
\dot{x}_{n} & =-\lambda_{n} x_{n}-\cdots-\lambda_{i+1} x_{i+1}+v_{i}
\end{aligned}
$$

from the input $v_{i}$ to the output $y_{i}=x_{i}$ will have one eigenvalue at the origin, $n-i$ eigenvalues on the left half complex plane and no zeros. Using the root locus we can see that for a small enough positive $\lambda_{i}$, the feedback $v_{i}=-\lambda_{i} x_{i}$ will achieve exponential stability. Finally, by a trivial property of the geometric series, if $w=0$

$$
|u| \leq \frac{\epsilon}{2}+\frac{\epsilon}{4}+\frac{\epsilon}{8}+\cdots+\frac{\epsilon}{2^{n}}+\frac{\epsilon}{2^{n+1}}<\epsilon
$$

and $\epsilon$ can be arbitrarily selected.

Remark 12: The design option that the saturation levels should follow the geometric series $(\epsilon / 2),(\epsilon) /\left(2^{2}\right), \ldots,(\epsilon) /\left(2^{n}\right)$ is academic, namely it is considered for the case of an infinite chain of integrators because of the property (57). In practical situations, one can use the feedback

$u=-\epsilon_{n} \sigma_{s}\left(\frac{\lambda_{n}}{\epsilon_{n}} x_{n}\right)-\cdots-\epsilon_{2} \sigma_{s}\left(\frac{\lambda_{2}}{\epsilon_{2}} x_{2}\right)-\epsilon_{1} \sigma_{s}\left(\frac{\lambda_{1}}{\epsilon_{1}} x_{1}\right)$

where, if $u_{\max }$ is the maximum available control energy, the constants $\epsilon_{1}, \epsilon_{2}, \ldots, \epsilon_{n}$ must be such that

$$
\begin{aligned}
\epsilon_{n} & >\epsilon_{n-1}+\cdots+\epsilon_{2}+\epsilon_{1} \\
& \vdots \\
\epsilon_{2} & >\epsilon_{1} \\
\epsilon_{1} & >0 \\
u_{\max } & \geq \epsilon_{n}+\cdots+\epsilon_{2}+\epsilon_{1} .
\end{aligned}
$$

The feasibility of the aforementioned system of inequalities is trivial, since we know at least one solution, for example, $\epsilon_{i}=$ (1) $/\left(2^{n-i+1}\right) u_{\max }$. Replacing the last inequality in (58) with the equality constraint $\epsilon_{n}+\cdots+\epsilon_{2}+\epsilon_{1}=u_{\max }$ we can treat the problem of finding the appropriate set of $\epsilon_{i}$ as an optimization

\footnotetext{
${ }^{11}$ In fact, the lower subsystem of (56) is ISS with restrictions. See also Remark
}

problem. This approach allows us to increase the saturation level in the feedback of the upper component $x_{1}$ enhancing the overall performance of the closed-loop system.

Remark 13: System (54) is a special case of the class of systems studied in [19]. Therein, a similar construction has been performed. However, in the proposed design the saturating gains, namely $(\epsilon / 2),(\epsilon / 4), \ldots$, are constants, whereas in [19] the gains are functions of the state and have to satisfy some nontrivial conditions. Finally, for large values of $\|x\|$, the saturating gains in [19] tend to zero, and this is not the case for the control law (55).

The result in Proposition 4 can be easily extended to a larger class of systems, namely nonlinear chains of integrators described by equations of the form

$$
\begin{aligned}
\dot{x}_{1} & =\phi_{1}\left(x_{2}\right) \\
& \vdots \\
\dot{x}_{n-1} & =\phi_{n-1}\left(x_{n}\right) \\
\dot{x}_{n} & =\phi_{n}(u)
\end{aligned}
$$

with $d \phi_{i}(0)>0$, for all $i=1, \ldots, n$. For illustration purposes, consider the system described by the equations

$$
\begin{aligned}
& \dot{x}_{1}=\sin \left(x_{2}\right) \quad \dot{x}_{2}=\sin \left(x_{3}\right) \quad \dot{x}_{3}=\sin \left(x_{4}\right) \\
& \dot{x}_{4}=\sin (u) .
\end{aligned}
$$

In Fig. 3, the response of (60) in closed loop with

$$
\begin{aligned}
u=-\frac{\epsilon}{2} \sigma_{s}\left(\frac{2 \lambda_{4}}{\epsilon}\right. & \left.x_{4}\right)-\frac{\epsilon}{4} \sigma_{s}\left(\frac{4 \lambda_{3}}{\epsilon} x_{3}\right) \\
& -\frac{\epsilon}{8} \sigma_{s}\left(\frac{8 \lambda_{2}}{\epsilon} x_{2}\right)-\frac{\epsilon}{16} \sigma_{s}\left(\frac{16 \lambda_{1}}{\epsilon} x_{1}\right)
\end{aligned}
$$

is presented. For this particular case, global asymptotic stability can be achieved if $\epsilon \in\left(0, \epsilon^{\star}\right]$ with $\epsilon^{\star}<(\pi / 2)$. In the particular simulations, we use $\epsilon=(\pi / 4)$ and $\left[\lambda_{1}, \lambda_{2}, \lambda_{3}, \lambda_{4}\right]=$ $[0.008,0.108,0.540,1.20]$.

Remark 14: Output feedback stabilization of (54) with output $\eta=x_{1}$ can be addressed by a straightforward application of Proposition 4 and [33, Th. 7.1]. Finally, chains of integrators of the form

$$
\dot{x}_{1}=\alpha_{1} x_{2}, \ldots, \dot{x}_{n-1}=\alpha_{n-1} x_{n} \quad \dot{x}_{n}=\alpha_{n} u
$$

where $0<\underline{\alpha}_{j} \leq \alpha_{j} \leq \bar{\alpha}_{j}, j=1, \ldots, n$ and the limits $\underline{\alpha}_{j}, \bar{\alpha}_{j}$ are known, can be treated following the steps of the proof of Proposition 4. Robust stabilization of (62) in the presence of uncertain system parameters has also been studied in [18]. The nested saturation scheme employed there also required some nontrivial algebraic conditions to be satisfied.

\section{B. Asymptotic Stabilizability by Control of Constant Sign}

In this section, we present a general result on the asymptotic stabilizability of linear stable systems with bounded control of constant sign, that is a consequence of Proposition 2 or Corollary 2.

Proposition 5: Any stable and controllable linear system

$$
\dot{x}=A x+B u
$$



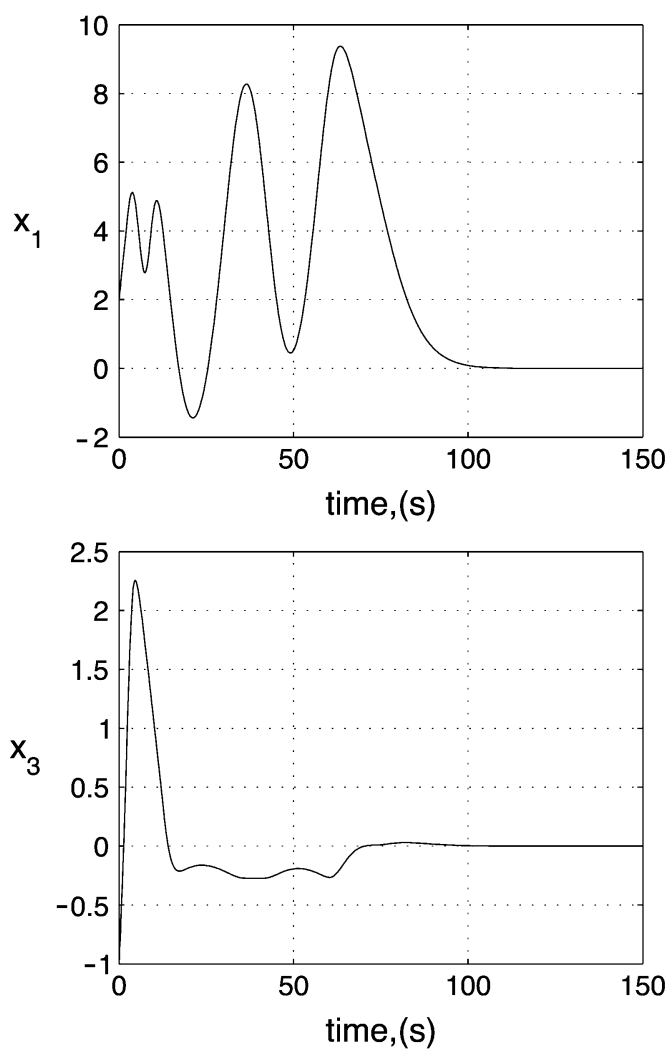

Fig. 3. State histories of the closed-loop system (60)-(61).

with $A$ such that $\operatorname{det}(A) \neq 0$, is asymptotically stabilizable by positive (or negative) control.

Proof: Note first that because $\operatorname{det}(A) \neq 0$, the matrix $A$ has no zero eigenvalue. It can be verified that, under the assumptions of Proposition 5, (63) can be written, in a set of suitable coordinates, in the form

$$
\begin{aligned}
& \dot{z}=J z+H \xi+R u \\
& \dot{\xi}=F \xi+G u
\end{aligned}
$$

where $z \in \mathbb{R}^{p}, \xi \in \mathbb{R}^{m}, p+m=n$, and $J+J^{\prime} \leq 0, \operatorname{det}(J) \neq 0$. The last equation of the cascade (64) represents the asymptotically stable part of (63), if there is any, i.e., $F$ is Hurwitz. In the case where such an asymptotically stable part does not exist, it is easy to verify that the system $\dot{z}=J z+R u$ is globally asymptotically stabilized by the control law

$$
u=-\frac{\epsilon}{2} \sigma_{+}\left(\frac{2}{\epsilon} K z\right) \quad\left(\text { or } \quad u=\frac{\epsilon}{2} \sigma_{+}\left(-\frac{2}{\epsilon} K z\right)\right)
$$

for all $\epsilon>0$ and for some appropriately chosen ${ }^{12} K \in \mathbb{R}^{1 \times p}$. According to Corollary 2, a similar control law ${ }^{13}$ would also stabilize the cascade (64) if the asymptotically stable part exists. $\triangleleft$

Remark 15: The results in Proposition 5 and Corollary 2 should be examined in the light of what established in [28], where it was proven that a linear system $\dot{x}=A x+B u$ is locally controllable at the origin with $u(t) \in[0,1]$, for all $t$, if and only if the pair $\{A, B\}$ is controllable in the ordinary sense and

\footnotetext{
${ }^{12}$ In fact, in this case a "good" saturated linear feedback can be obtained by invoking standard passivity arguments.

${ }^{13}$ Not necessarily with the same $K$.
}
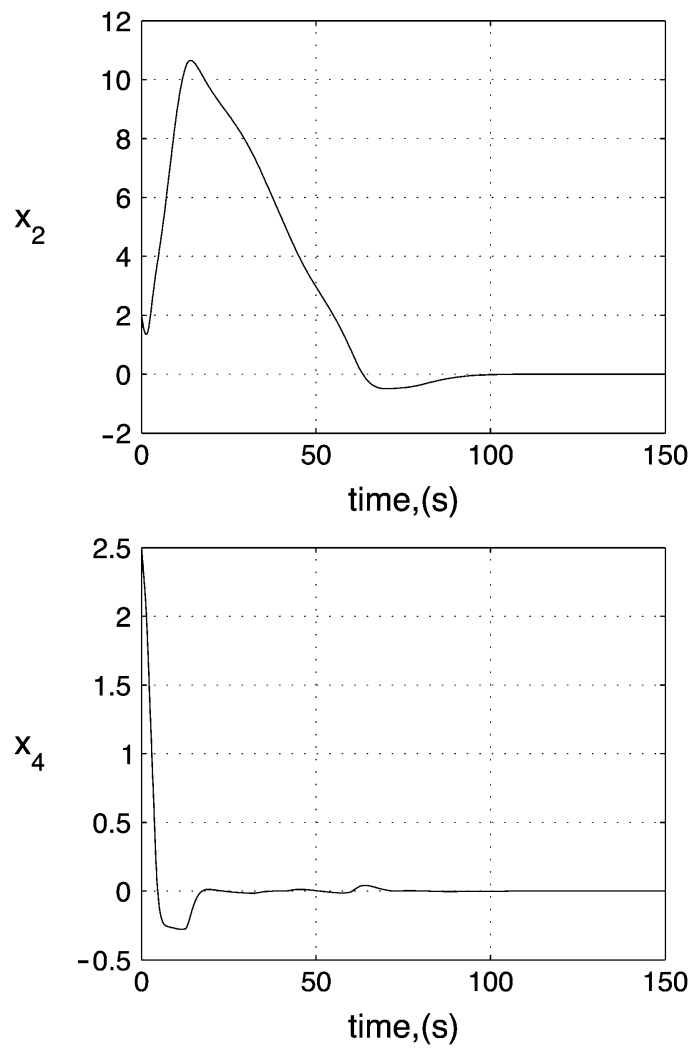

all eigenvalues of $A$ have nonzero imaginary parts. Using [28] and [4], it is easy to show that a linear system is asymptotically controllable with positive (or negative) bounded control if and only if $\operatorname{spec}(A) \subset \mathbf{C}^{-} \cup\left\{\mathbf{C}^{0} \backslash\{0\}\right\}$.

\section{Asymptotic Stabilization of the TORA}

In this section, we apply the results of Section IV to solve the asymptotic stabilization problem for the TORA [3]. After appropriate normalizing transformations [37], the dynamics of the system are described by the equations

$$
\begin{aligned}
\ddot{x}_{d}+x_{d} & =\gamma\left(\dot{\phi}^{2} \sin \phi-\ddot{\phi} \cos \phi\right) \\
\ddot{\phi} & =u-\gamma \ddot{x}_{d} \cos \phi
\end{aligned}
$$

where $x_{d}$ is the translational position, $v_{d}=\dot{x}_{d}$ the translational velocity, $\phi$ the angular position, $\omega=\dot{\phi}$ the angular velocity and $0<\gamma<1$ a constant depending on the physical parameters of the device. The presence of the term in $\omega^{2}$ in the model makes the stabilization of the system an intricate problem, especially considering that an ideal control law would utilize measurements of the translational and angular positions only. It is shown in [11] that, via a coordinates transformation, (66) can be written in the form

$$
\begin{aligned}
& \dot{x}_{1}=x_{2} \\
& \dot{x}_{2}=-x_{1}+\gamma \sin x_{3} \\
& \dot{x}_{3}=\frac{1}{\psi\left(x_{3}\right)} x_{4} \\
& \dot{x}_{4}=\frac{\psi\left(x_{3}\right)}{1-\gamma^{2} \cos ^{2} x_{3}} u+\frac{\gamma\left(x_{1}-\gamma \sin x_{3}\right) \cos x_{3}}{1-\gamma^{2} \cos ^{2} x_{3}} \psi\left(x_{3}\right)
\end{aligned}
$$


where

$$
\psi\left(x_{3}\right)=-\sqrt{\frac{1-\gamma^{2} \cos ^{2} x_{3}}{1-\gamma^{2}}}
$$

The measured variables are $x_{1}$ and $x_{3}$, which are functions of the translational and angular positions only. In [11], global output feedback stabilization and tracking was achieved with a combination of a nonlinear observer and backstepping. Here, we propose a simpler output feedback scheme. First, consider the preliminary feedback transformation

$u=u\left(v, x_{1}, x_{3}\right)=\frac{1-\gamma^{2} \cos ^{2} x_{3}}{\psi\left(x_{3}\right)} v-\gamma\left(x_{1}-\gamma \sin x_{3}\right) \cos x_{3}$

and the subsystem

$$
\begin{aligned}
& \dot{x}_{3}=\alpha\left(x_{3}\right) x_{4} \\
& \dot{x}_{4}=v
\end{aligned}
$$

with $\alpha\left(x_{3}\right)=(1) /\left(\psi\left(x_{3}\right)\right)$ and output $y=x_{3}$. Stabilization of (69) can be achieved using the dynamic output feedback

$$
\begin{aligned}
\dot{\hat{\theta}}= & -\left(\lambda\left(x_{3}\right)+1\right) \hat{\theta}-\left(\lambda\left(x_{3}\right)+1\right) \beta\left(x_{3}\right) \\
& -x_{3} \alpha\left(x_{3}\right)+(b+1) w \\
v= & -x_{3} \alpha\left(x_{3}\right)-\hat{\theta}-\beta\left(x_{3}\right)+w
\end{aligned}
$$

where $w$ is a new control variable to be used in the next step, $\beta\left(x_{3}\right)$ and $\lambda\left(x_{3}\right)$ are functions satisfying

$$
\frac{\partial \beta\left(x_{3}\right)}{\partial x_{3}} \alpha\left(x_{3}\right)=\lambda\left(x_{3}\right) \quad \lambda\left(x_{3}\right)>0
$$

and $b$ is a constant to be selected later. Asymptotic stabilization can be proven considering the new coordinate $z=\hat{\theta}+\beta\left(x_{3}\right)-$ $x_{4}$, and noting that (69)-(70) can be written as

$$
\begin{aligned}
\dot{x}_{3} & =\alpha\left(x_{3}\right) x_{4} \\
\dot{x}_{4} & =-x_{3} \alpha\left(x_{3}\right)-x_{4}-z+w \\
\dot{z} & =-\lambda\left(x_{3}\right) z+b w
\end{aligned}
$$

which is LES-ISS. Condition (71) can be seen either as a differential equation for the definition of $\beta\left(x_{3}\right)$, if $\lambda\left(x_{3}\right)$ is selected by the designer, or as the definition of $\lambda\left(x_{3}\right)$, if $\beta\left(x_{3}\right)$ is selected. For example, $\beta\left(x_{3}\right)=-\beta x_{3}$ with $\beta>0$ is a simple choice. Consider now the cascade

$$
\begin{aligned}
\dot{x}_{1} & =x_{2} \\
\dot{x}_{2} & =-x_{1}+\gamma \sin x_{3} \\
\dot{x}_{3} & =\alpha\left(x_{3}\right) x_{4} \\
\dot{x}_{4} & =-x_{3} \alpha\left(x_{3}\right)-x_{4}-z+w \\
\dot{z} & =-\lambda\left(x_{3}\right) z+b w
\end{aligned}
$$

that results from the first two of (67) and (72) and note that it satisfies Assumptions A1) and A2), hence can be asymptotically stabilized with bounded control of the form

$$
w=-\epsilon \sigma_{s}\left(\frac{1}{\epsilon} K\left[\begin{array}{l}
x_{1} \\
x_{2}
\end{array}\right]\right)
$$

with $K$ selected as in Lemma $1 .{ }^{14}$ However, to obtain an output feedback controller, $K$ has to be of the form $K=\left[\begin{array}{ll}k_{1} & 0\end{array}\right]$, for some $k_{1}$. We now show that such a $K$ exists. For, consider the approximation of (73) for small $\left\|\left[\begin{array}{lll}x_{3} & x_{4} & z\end{array}\right]^{\prime}\right\|$, and define the matrices

$$
\begin{aligned}
& J=\left[\begin{array}{cc}
0 & 1 \\
-1 & 0
\end{array}\right] \quad H=\left[\begin{array}{lll}
0 & 0 & 0 \\
\gamma & 0 & 0
\end{array}\right] \\
& F=\left[\begin{array}{ccc}
0 & \alpha & 0 \\
-\alpha & -1 & -1 \\
0 & 0 & -\lambda
\end{array}\right] \quad G=\left[\begin{array}{l}
0 \\
1 \\
b
\end{array}\right]
\end{aligned}
$$

where $\alpha=\alpha(0)$ and $\lambda=\lambda(0)$. From Lemma 1

$$
K=\chi G^{\prime} \bar{Y}=\chi\left[\bar{Y}_{1,2}+b \bar{Y}_{1,3} \quad \bar{Y}_{2,2}+b \bar{Y}_{2,3}\right]
$$

where $\chi$ is a positive constant, $\bar{Y}$ is the matrix that solves the Sylvester equation $H+\bar{Y} F+J^{\prime} \bar{Y}=0$, and $\bar{Y}_{i, j}$ is the $(i, j)$ entry of $\bar{Y}$. Selecting $b=-\left(\bar{Y}_{2,2}\right) /\left(\bar{Y}_{2,3}\right)$ yields $K=\left[\begin{array}{ll}k_{1} & 0\end{array}\right]$, hence, $w=w\left(x_{1}\right)$. This is possible, as it can be shown that with $\gamma \neq 0$ and $\alpha=-1, \bar{Y}_{2,3} \neq 0$ for all $\lambda$. The result is summarized in the following statement.

Proposition 6: Consider system (67) and a nonlinearity $\sigma(\cdot)=\sigma_{s}(\cdot)$ or $\sigma(\cdot)=\sigma_{+}(\cdot)$, belonging to the sector $[0,1]$. There exist constants $b, k_{1}, \epsilon \in \mathbb{R}$, with $\epsilon>0$ and a positive function $\lambda\left(x_{3}\right)$ such that system (67) in closed loop with the dynamic output feedback controller

$$
\begin{aligned}
\dot{\hat{\theta}}= & -(\lambda+1) \hat{\theta}-(\lambda+1) \beta\left(x_{3}\right) \\
& -x_{3} \alpha\left(x_{3}\right)-(b-1) \epsilon \sigma\left(\frac{1}{\epsilon} k_{1} x_{1}\right) \\
v= & -x_{3} \alpha\left(x_{3}\right)-\hat{\theta}-\beta\left(x_{3}\right)-\epsilon \sigma\left(\frac{1}{\epsilon} k_{1} x_{1}\right) \\
u= & \frac{1-\gamma^{2} \cos ^{2} x_{3}}{1-\psi\left(x_{3}\right)} v-\gamma\left(x_{1}-\gamma \sin x_{3}\right) \cos x_{3}
\end{aligned}
$$

is GAS (LES).

The control law (76) is much simpler in structure and implementation than the output feedback designs proposed in [11] or [22], while in [29] only state feedback is considered. In Fig. 4, some simulation results of the closed loop with the proposed controller are depicted. For the simulations we have used, as in [29], $\gamma=0.1$, so that the results are directly comparable with the ones given in this reference. It can be concluded that full-state feedback does not outperform the output feedback presented here.

\section{Stabilization of Underactuated Ships on a Linear Course}

In this section, we apply the result of Proposition 2 for the global asymptotic stabilization of a normalized model of an underactuated ship moving on a linear course. The model examined is taken from [5], were the authors designed state and output feedback controllers based on the backstepping technique and nonlinear observers. Their controllers achieve global

\footnotetext{
GAS.
} 

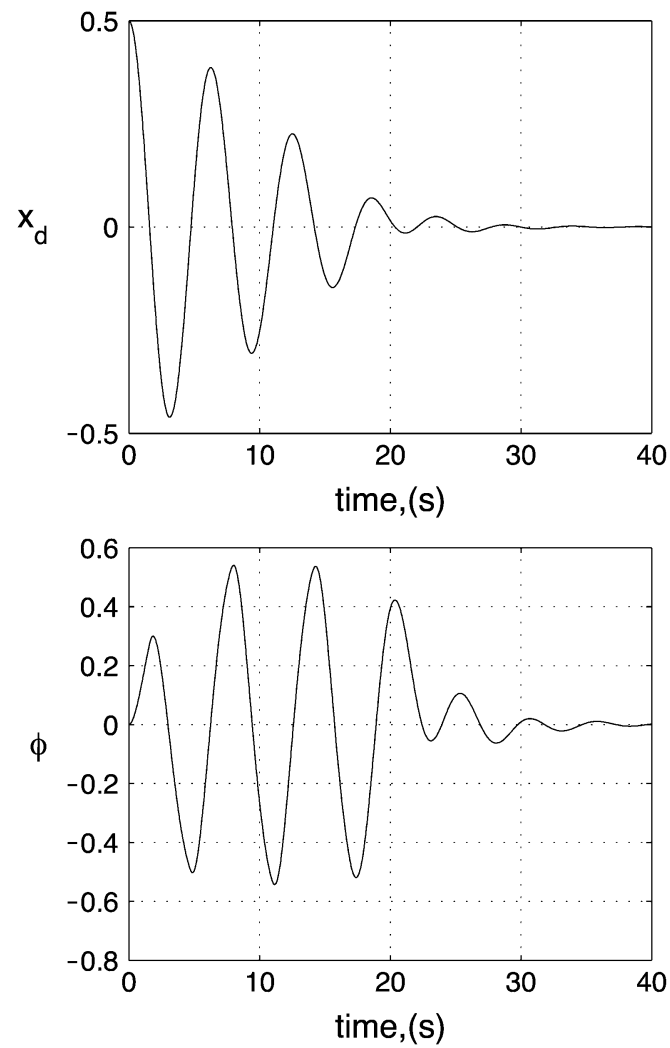

Fig. 4. State histories of the closed-loop system (67)-(76).

tracking of a straight line in the presence of nonvanishing environmental disturbances, that occur due to wave, wind and ocean current. Such a model is given by

$$
\begin{aligned}
\dot{y} & =u \sin (\psi)+\cos (\psi) \nu \\
\dot{\psi} & =r \\
\dot{\nu} & =-\frac{m_{1} u}{m_{2}} r-\frac{d_{2}}{m_{2}} \nu-\frac{d_{\nu 2}}{m_{2}}|\nu| \nu+\frac{1}{m_{2}} \tau_{w \nu}(t) \\
\dot{r} & =\frac{\left(m_{1}-m_{2}\right) u}{m_{3}} \nu-\frac{d_{3}}{m_{3}} r-\frac{d_{r 2}}{m_{2}}|r| r+\frac{1}{m_{3}} \tau_{r}+\frac{1}{m_{3}} \tau_{w r}(t)
\end{aligned}
$$

where $y, \nu$ are the sway displacement (deviation from the course on the axis vertical to the ship axis) and velocity and $\psi, r$ are the yaw angle and velocity. The forward speed, that is controlled independently by the main thruster control system, is given by $u$, and is considered constant, or slowly varying. The control action is represented by $\tau_{r}$, the torque applied to the ship rudder. The positive constants $m_{i}, i=1,2,3$ denote the ship inertia with respect to the three axis, including added mass, and the positive constants $d_{2}, d_{3}, d_{\nu 2}, d_{r 2}$ denote the hydrodynamic damping in sway and yaw. The terms $\tau_{w \nu}(t), \tau_{w r}(t)$ represent the environmental disturbance moments and are considered to be bounded.

System (77) is in block feedforward form, i.e., we can distinguish the interconnection of the subsystem of $\left[\begin{array}{ll}\nu & r\end{array}\right]^{\prime}$ with the integrator $\dot{\psi}=r$ and, at the next step, the interconnection of the subsystem of $\left[\begin{array}{lll}\psi & \nu & r\end{array}\right]^{\prime}$ with the subsystem $\dot{y}=$ $u \sin (\psi)+\cos (\psi) \nu$. The inertia, $m_{2}$, around the second axis of the ship is always larger than the inertia, $m_{1}$, around the first axis which implies that the linear part of the subsystem of $\left[\begin{array}{ll}\nu & r\end{array}\right]^{\prime}$
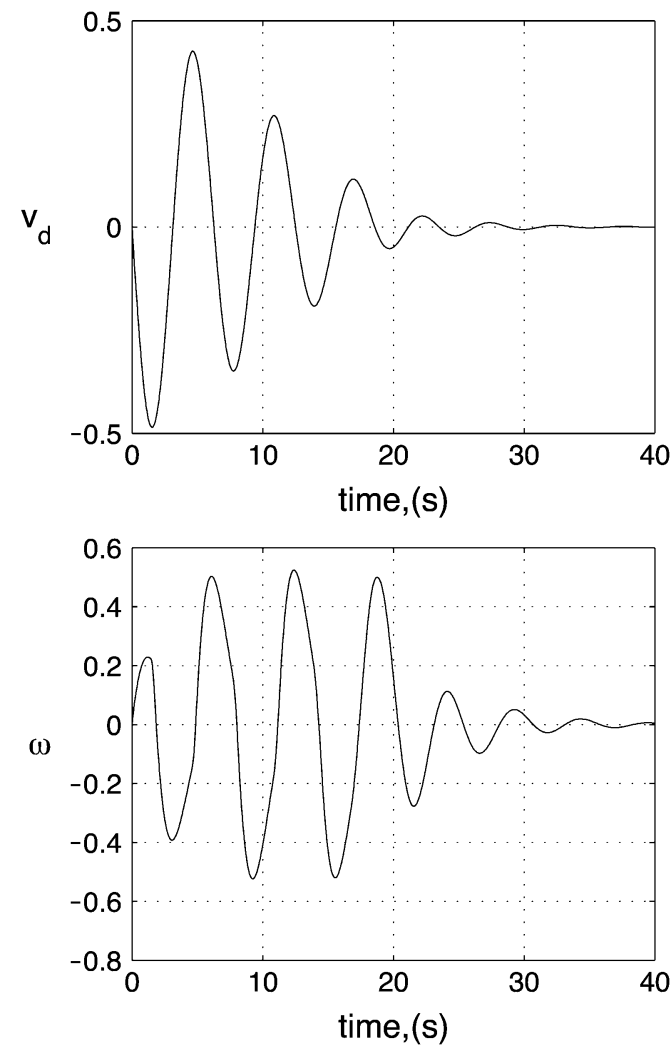

is exponentially stable $\left(m_{1}-m_{2}<0\right)$. In addition, the nonlinear damping terms $-\left(d_{\nu 2}\right) /\left(m_{2}\right)|\nu| \nu$ and $-\left(d_{r 2}\right) /\left(m_{2}\right)|r| r$ do not "disturb" this stability property, so it is easy to verify that all assumptions of Proposition 2 are satisfied for the cascade of the subsystem of $\left[\begin{array}{ll}\nu & r\end{array}\right]^{\prime}$ with the integrator $\dot{\psi}=r$. For such a system, a bounded control law, feeding back $\psi$ only, can be designed. Next, repeating the procedure once more, we obtain a stabilizing controller for the cascade (77), namely

$$
\tau_{r}=-\frac{\epsilon}{2} \sigma_{s}\left(\frac{2 \lambda_{1}}{\epsilon} \psi\right)-\frac{\epsilon}{2.1} \sigma_{s}\left(\frac{2.1 \lambda_{2}}{\epsilon} y\right)
$$

where $\lambda_{1}$ and $\lambda_{2}$ are suitably chosen. Note that we have used the arguments in Remark 12 to enhance the performance of the controller. To illustrate the properties of the closed-loop system (77)-(78) via simulations we consider a simplified situation where $m_{1}=1, m_{2}=2, m_{3}=1, d_{2}=2, d_{3}=2, d_{\nu 2}=$ $0.1, d_{r 2}=0.1, \tau_{w \nu}(t)=0$, and $\tau_{w r}(t)=0$, and the nominal forward speed is $u=1$. For this set of parameters, appropriate gains for the controller (78) are $\left(\lambda_{1}, \lambda_{2}\right)=(1.4,0.86)$ and $\epsilon=5$. In Fig. 5 we depict the state histories and the control action of the closed-loop system (77)-(78).

\section{CONCLUSION}

The problem of stabilization of a class of cascaded systems with bounded control has been addressed and solved using the linear bounded real lemma and a generalized version of the small gain theorem. Globally asymptotically stabilizing control laws that require only partial state feedback have been designed. These control laws make use of typical saturating functions, constant sign saturations or quantizations and they exhibit 

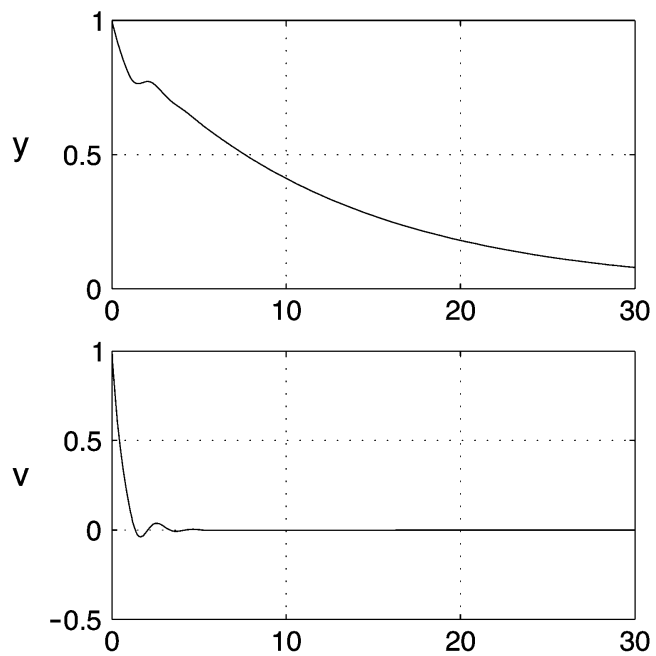
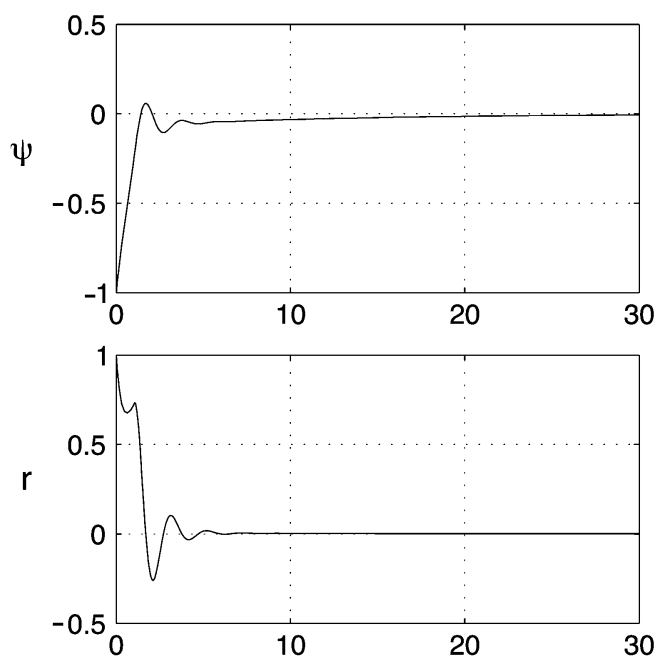

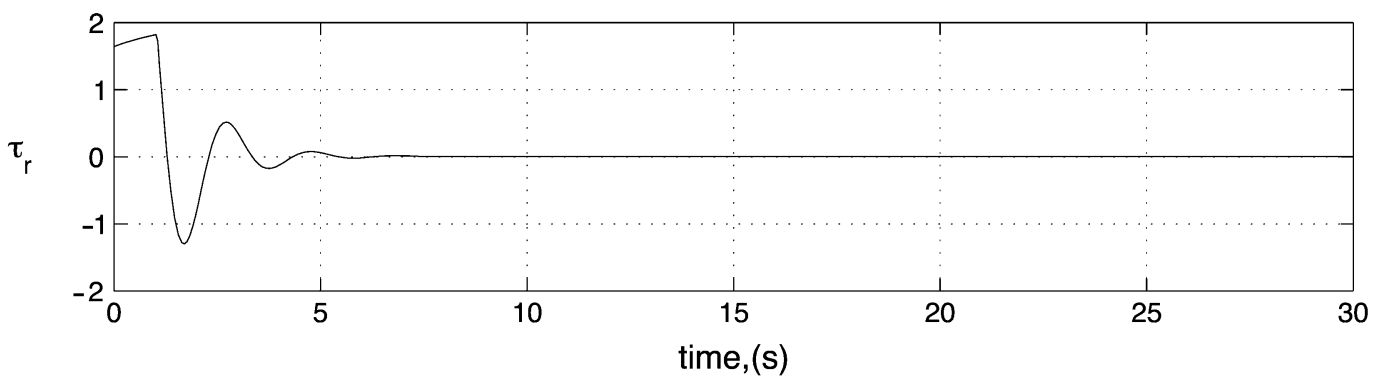

Fig. 5. State histories and control action of the closed-loop system (77)-(78).

a simple structure, however, in some cases, they require the amplitude of the control signal to be kept small enough.

The main results are applied to the global stabilization problem for a chain of integrators subject to input saturation, yielding a control law that is significantly different from existing results and also to the global stabilization of the nonlinear benchmark system of TORA and to the stabilization of underactuated ships moving on a linear course. At the same time, the new stabilization scheme provides motivation for a dynamic output feedback stabilization methodology, which can accommodate saturated outputs. This dynamic solution is clearly different from observation-based schemes available in the literature.

\section{ACKNOWLEDGMENT}

The authors would like to thank Prof. T. Iwasaki of University of Virginia for his help on the Proof of Lemma 1, Prof. L. Praly of Ecole des Mines de Paris, for his comments on an early version of this paper, and the Associate Editor and the anonymous reviewers for several constructive remarks.

\section{REFERENCES}

[1] M. Arcak, A. R. Teel, and P. V. Kokotović, "Robust nonlinear control of feedforward systems with unmodeled dynamics," Automatica, vol. 37, pp. 265-272, 2001.

[2] A. Astolfi, R. Ortega, and R. Sepulchre, "Passivity-based control of nonlinear systems," in Control of Complex Systems, K. Astrom, P. Albertos, M. Blanke, A. Isidori, W. Schaufelberger, and R. Sanz, Eds. New York: Springer-Verlag, 2001.
[3] R. T. Bupp and D. S. Bernstein, "A benchmark problem for nonlinear control design: Problem statement, experimental testbed, and passive nonlinear compensation," in Proc. Amer. Control Conf., 1995, pp. 4363-4367.

[4] P. DeLeenheer and D. Aeyels, "Stabilization of positive linear systems," Syst. Control Lett., vol. 44, no. 4, pp. 259-271, 2001.

[5] K. D. Do, Z. P. Jiang, and J. Pan, "Robust global stabilization of underactuated ships on a linear course: State and output feedback," Int. J. Control, vol. 76, no. 1, pp. 1-17, 2003.

[6] R. Freeman, "Time-varying feedback for the global stabilization of nonlinear systems with measurement disturbances," presented at the Eur. Control Conf., Brussels, Belgium, 1997.

[7] G. Garcia and S. Tarbouriech, "Stabilization with eigenvalues placement of a norm bounded uncertain system by bounded inputs," Int. J. Robust Nonlinear Control, vol. 9, pp. 599-615, 1999.

[8] M. Green and D. J. N. Limebeer, Linear Robust Control. Upper Saddle River, NJ: Prentice-Hall, 1995.

[9] F. Grognard, R. Sepulchre, and G. Bastin, "Global stabilization of feedforward systems with exponentially unstable Jacobian linearization," Syst. Control Lett., vol. 37, no. 2, pp. 107-115, 1999.

[10] A. Isidori, Nonlinear Control Systems II. New York: Springer-Verlag, 1999.

[11] Z.-P. Jiang and I. Kanellakopoulos, "Global output-feedback tracking for a benchmark nonlinear system," IEEE Trans. Automat. Contr., vol. 45, pp. 1023-1027, May 2000.

[12] G. Kaliora, "Control of nonlinear systems with bounded signals," $\mathrm{Ph} . \mathrm{D}$. dissertation, Imperial College, Univ. London, London, U.K., 2002.

[13] G. Kaliora and A. Astolfi, "Output feedback asymptotic stabilization with bounded measurements," in Proc. 4th Asian Control Conf., 2002, pp. 857-862.

[14] G. Kreisselmeier, "Stabilization of linear systems in the presence of output measurement saturation," Syst. Control Lett., vol. 29, no. 1, pp. 27-30, 1996.

[15] Y. Lin and E. D. Sontag, "A universal formula for stabilization with bounded controls," Syst. Control Lett., vol. 16, pp. 393-397, 1991.

[16] Z. Lin and T. Hu, "Semi-global stabilization of linear systems subject to output saturation," Syst. Control Lett., vol. 43, no. 3, pp. 211-217, 2001.

[17] Z. Lin and A. Saberi, "Semi-global exponential stabilization of linear systems subject to "input saturation" via linear feedbacks," Syst. Control Lett., vol. 21, pp. 225-239, 1993. 
[18] L. Marconi and A. Isidori, "Robust global stabilization of a class of uncertain feedforward nonlinear systems," Syst. Control Lett., vol. 41, no. 4, pp. 281-290, 2000.

[19] F. Mazenc, "Stabilization of feedforward systems approximated by a nonlinear chain of integrators," Syst. Control Lett., vol. 32, no. 4, pp. 223-229, 1997.

[20] F. Mazenc, S. Mondie, and S.-I. Niculescu, "Global asymptotic stabilization for chains of integrators with a delay in the input," in Proc. 40th IEEE Conf. Decision and Control, 2001, pp. 1843-1848.

[21] F. Mazenc and L. Praly, "Adding integrations, saturated controls and stabilization for feedforward systems," IEEE Trans. Automatic Control, vol. 41, pp. 1559-1578, Nov. 1996.

[22] F. Mazenc and J. C. Vivalda, "Global asymptotic output feedback stabilization of feedforward systems," in Proc. Eur. Control Conf., 2001, pp. 2564-2569.

[23] D. Nesic and E. Sontag, "Output stabilization of nonlinear systems: Linear systems with positive outputs as a case study," in Proc. 37th IEEE Conf. Decision and Control, 1998, pp. 885-890.

[24] R. Ortega, A. Loria, P. J. Nicklasson, and H. Sira-Ramirez, Passivity-Based Control of Euler-Lagrange Systems. New York: Springer-Verlag, 1998.

[25] L. Praly, R. Ortega, and G. Kaliora, "Stabilization of nonlinear systems via forwarding $\bmod \left\{\mathrm{L}_{\mathrm{g}} \mathrm{V}\right\}$," IEEE Trans. Automat. Contr., vol. 46, pp. 1461-1466, Sept. 2001.

[26] A. Rapaport and A. Astolfi, "A remark on the stability of interconnected nonlinear systems," IEEE Trans. Automat. Contr., vol. 49, pp. 120-124, Jan. 2004.

[27] A. Saberi, J. Han, and A. Stoorvogel, "Constrained stablization problems for linear plants," Automatica, vol. 38, pp. 639-654, 2002.

[28] S. Saperstone and J. Yorke, "Controllability of linear oscillatory systems using positive controls," in SIAM J. Control, vol. 9, 1971, pp. 253-262.

[29] R. Sepulchre, M. Jankovic, and P. V. Kokotoviæ, Constructive Nonlinear Control. New York: Springer-Verlag, 1996.

[30] E. Sontag, "Further facts about input to state stabilization," IEEE Trans. Automat. Contr., vol. 35, pp. 473-476, Apr. 1990.

[31] - "The ISS philosophy as a unifying framework for stability-like behavior," in Nonlinear Control in the Year 2000, A. Isidori, F. Lamnabhi, and W. Respondek, Eds. London, U.K.: Springer-Verlag, 2001.

[32] A. Stoorvogel and A. Saberi, "Output regulation for linear plants with actuators subject to amplitude and rate constraints," Int. J. Robust Nonlinear Control, vol. 9, pp. 631-657, 1999.

[33] H. Sussmann, E. Sontag, and Y. Yang, "A general result on the stabilization of linear systems using bounded controls," IEEE Trans. Automat. Contr., vol. 39, pp. 2411-2425, Dec. 1994.

[34] M. Sznaier, R. Suarez, S. Miani, and J. Alvarez-Ramirez, "Optimal $l^{\infty}$ disturbance attenuation and global stabilization of linear systems with bounded control," Int. J. Robust Nonlinear Control, vol. 9, pp. 659-675, 1999.

[35] A. R. Teel, "Global stabilization and restricted tracking for multiple integrators with bounded controls," Syst. Control Lett., vol. 18, pp. 165-171, 1992.
[36] A. R. Teel, "A nonlinear small gain theorem for the analysis of control systems with saturation," IEEE Trans. Automat. Contr., vol. 41, pp. 1256-1270, Sept. 1996.

[37] C.-J. Wan, D. S. Bernstein, and V. T. Coppola, "Global stabilization of the oscillating eccentric rotor," Nonlinear Dyna., vol. 10, pp. 49-62, 1996.

[38] J. L. Willems, Stability Theory of Dynamical Systems. New York: Nelson, 1970.

[39] W. M. Wonham, Linear Multivariable Control. New York: SpringerVerlag, 1995.

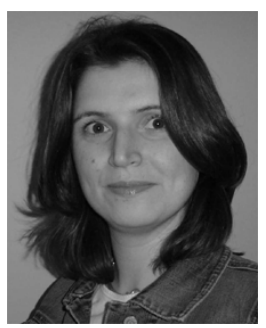

Georgia Kaliora was born in Athens, Greece, in 1974. She received the M.Eng. degree in mechanical engineering from the National Technical University of Athens, Athens, Greece, in 1997, and the Ph.D. degree from the University of London, Imperial College, London, U.K., for her work on the stabilization of nonlinear systems with bounded signals, in 2002 .

Between November 2002 and March 2004, she worked as a Research Associate in the Department of Electrical and Electronic Engineering, Imperial College London, continuing her work on constructive nonlinear control and on the existence and construction of nonlinear observers.

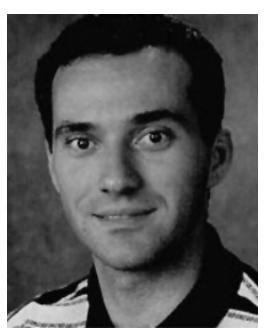

Alessandro Astolfi was born in Rome, Italy, in 1967. He graduated in electronic engineering from the University of Rome "La Sapienza," Rome, Italy, in 1991, and received the Ph.D. degree (with Medal of Honor) in information theory from ETH-Zurich, Zurich, Switzerland, in 1995, and the Ph.D. degree from the University of Rome "La Sapienza," in 1996.

Since 1996, he has been with the Department of Electrical and Electronic Engineering, Imperial College, London, U.K., where he is currently a Reader in nonlinear control theory. From 1998 to 2003, he was also an Associate Professor with the Department of Electronics and Information of the Politecnico of Milan, Milan, Italy. His research interests are focused on mathematical control theory and control applications, with special emphasis on the problems of discontinuous stabilization, robust stabilization, robust control, and adaptive control. He is the author of more than 30 journal papers, ten book chapters, and over 100 papers in refereed conference proceedings. He is an Associate Editor of Systems and Control Letters, Automatica, the International Journal of Control, and the European Journal of Control. He has also served on the IPC and NOC of various international conferences. 